\title{
Einfluss der Körperposition auf die Lungenfunktion: eine systematische Übersichtsarbeit
}

\author{
Shikma Katz ${ }^{\mathrm{a}, \mathrm{c}}$ Nissim Arish ${ }^{\mathrm{b}, \mathrm{d}} \quad$ Ariel Rokach $^{\mathrm{b}, \mathrm{d}}$ Yacov Zaltzmann ${ }^{\mathrm{a}}$ Esther-Lee Marcus ${ }^{\mathrm{a}, \mathrm{d}}$ \\ ${ }^{a}$ Chronic Ventilator-Dependent Division, Herzog Medical Center, Jerusalem, Israel; \\ bPulmonary Institute, Shaare Zedek Medical Center, Jerusalem, Israel; \\ ${ }^{\mathrm{C}}$ Recanati School for Community Health Professions, Faculty of Health Sciences, Ben Gurion University of the Negev, Beer Sheva, Israel; \\ ${ }^{\mathrm{d} H e b r e w}$ University-Hadassah Faculty of Medicine, Jerusalem, Israel
}

\section{Schlüsselwörter \\ Körperposition · Lungenvolumen · Physikalische Therapie · Positionierung · Körperhaltung · Lungenfunktion · Sitzend . Rückenlage $\cdot$ Stehend}

\section{Zusammenfassung}

Hintergrund: Die Lungenfunktionsprüfung (LFP) wird wegen der Anordnung der Messgeräte und des Patientenkomforts routinemäßig in aufrechter Position durchgeführt. In der vorliegenden systematischen Übersichtsarbeit wurde der Einfluss der Körperposition auf die Lungenfunktion bei gesunden Probanden und speziellen Patientengruppen untersucht.

Methoden: Zur Identifizierung englischsprachiger Publikationen, die zwischen Januar 1998 und Dezember 2017 veröffentlicht wurden, erfolgte eine Suche in MEDLINE und Google Scholar anhand der Suchbegriffe body position, lung function, lung mechanics, lung volume, position change, positioning, posture, pulmonary function testing, sitting, standing, supine, ventilation, and ventilatory change. Bei den eingeschlossenen Studien handelte es sich um quasi-experimentelle Prä-post-Interventionen, in denen mindestens 2 Positionen, einschließlich Sitzen oder Stehen, untersucht wurden und die die Lungenfunktion bei nicht mechanisch beatmeten Probanden $\geq 18$ Jahre bewerteten. Primäre Zielkriterien waren das forcierte exspiratorische Volumen in 1 Sekunde $\left(F E V_{1}\right)$, die forcierte Vitalkapazität (FVC, FEV $/$ FVC), die Vitalkapazität (VC), die funktionelle Residualkapazität (FRC), der maximale exspiratorische Druck (PEmax), der maximale inspiratorische Druck (PImax), der exspiratorische Spitzenfluss (PEF), die totale Lungenkapazität (TLC), das Residualvolumen (RV) und die Kohlenmonoxid-Diffusionskapazität der Lunge $\left(D_{\llcorner C O}\right)$. Folgende Positionen wurden untersucht: stehend, sitzend, Rückenlage sowie Rechts- und Linksseitenlage.
Ergebnisse: 43 Studien erfüllten die Einschlusskriterien. Die Studienpopulationen umfassten gesunde Probanden (29 Studien), Patienten mit Lungenerkrankungen (9), Herzkrankheiten (4), Rückenmarksverletzungen (SCI) (7), neuromuskulären Erkrankungen (3) und Adipositas (4). In den meisten Studien mit gesunden Probanden oder Patienten mit Lungen-, Herz- oder neuromuskulären Erkrankungen oder Adipositas fielen die Werte für FEV 1 , FVC, FRC, PEmax, PImax und/oder PEF in den aufrechteren Positionen höher aus. Bei Patienten mit SCl-bedingter Tetraplegie waren die Werte für FVC und FEV ${ }_{1}$ in Rückenlage höher als im Sitzen. Gesunde Probanden wiesen in Rückenlage eine höhere $D_{\text {Lco }}$ auf als im Sitzen und im Sitzen höhere Werte als in Seitenlage. Bei Patienten mit chronischer Herzinsuffizienz fiel der Einfluss der Körperposition auf die D Lco unterschiedlich aus.

Schlussfolgerungen: Die Körperposition hat Einfluss auf die Ergebnisse der LFP, doch bestehen Unterschiede hinsichtlich optimaler Position und Ausmaß des Benefits zwischen den Studienpopulationen. Die LFP erfolgt routinemäßig in sitzender Position. Wir empfehlen, bei Patienten mit Rückenmarksverletzung und neuromuskulären Erkrankungen neben der sitzenden Position auch die Rückenlage für die LFP in Betracht zu ziehen. Bei der Behandlung von Patienten mit Herz- oder Lungenerkrankungen, SCl, neuromuskulären Erkrankungen oder Adipositas ist zu berücksichtigen, dass die Physiologie und Funktion der Lunge von der Körperposition beeinflusst werden.

\footnotetext{
(c) 2019 Die Autoren
}

\section{KARGER \\ Fax +497614520714 \\ information@karger.com}

www.karger.com

\section{() 2019 S. Karger GmbH, Freiburg}

Accessible online at: www.karger.com/kkp
Dr. Ariel Rokach

Pulmonary Institute, Shaare Zedek Medical Center

POB 3235, Jerusalem, Israel

rokachariel@gmail.com 
- Mechanisch beatmete Patienten

- Sitzende oder stehende Position nicht beurteilt

- In die Übersichtsarbeit eingeschlossene Lungenfunktionsprüfung nicht beurteilt

- Kein statistischer Vergleich der Lungenfunktion in den relevanten Positionen

- Studienpopulation schloss Patienten $<18$ Jahre ein

\section{Hintergrund}

Die Lungenfunktionsprüfung (LFP) liefert objektive und quantifizierbare Maße der Lungenfunktion. Sie wird zur Beurteilung und Kontrolle von Erkrankungen, die die Herz- und Lungenfunktion beeinträchtigen, eingesetzt sowie zur Überwachung der Auswirkungen von umwelt- und berufsbedingten Belastungen und von Arzneimittel- bzw. Drogenexpositionen. Außerdem wird sie zur Abschätzung des Operationsrisikos und zur Beurteilung bei Einstellungsuntersuchungen sowie bei Untersuchungen aus versicherungsrechtlichen Gründen herangezogen. Die Spirometrie ist die häufigste Form der LFP [1]. Gemäß den Leitlinien der American Thoracic Society (ATS)/European Respiratory Society (ERS) kann die LFP im Sitzen oder im Stehen erfolgen und die Position sollte im Bericht vermerkt werden. Eine sitzende Position ist zum einen aus Sicherheitsgründen zu bevorzugen, um einen Sturz des Patienten infolge einer Synkope zu vermeiden [2], zum anderen weil sie für die Handhabung der Messgeräte und den Patienten unter Umständen bequemer ist. Allerdings kann es für Patienten, die an einer neuromuskulären Erkrankung, morbider Adipositas und anderen Krankheiten leiden, schwierig sein, während der Untersuchung zu sitzen oder zu stehen, was Auswirkungen auf die Ergebnisse haben kann.

Eines der Hauptziele der Positionierung und insbesondere der Untersuchung in aufrechten Positionen ist eine verbesserte Lungenfunktion von Patienten mit respiratorischen Erkrankungen, Herzinsuffizienz, neuromuskulären Erkrankungen, Rückenmarksverletzung (spinal cord injury, SCI) und Adipositas, und in den vergangenen 20 Jahren wurden zahlreiche Studien zum Einfluss der Körperposition auf die Atemmechanik und/oder -funktion veröffentlicht. Darunter war jedoch kein systematischer Review zu finden, der die Ergebnisse von Studien mit nicht mechanisch beatmeten Erwachsenen zusammenfasst, um daraus
Konsequenzen für die klinische Versorgung von Atemwegserkrankungen und die Durchführung der LFP abzuleiten.

Unser Ziel war eine systematische Überprüfung von Studien, in denen der Einfluss der Körperposition auf die Lungenfunktion bei gesunden Probanden und nicht mechanisch beatmeten Patienten mit Lungen- und Herzerkrankungen, SCI, neuromuskulären Erkrankungen und Adipositas beurteilt wurde.

\section{Methoden}

Zwei Wissenschaftlerinnen (S.K., E.-L.M.) durchsuchten MEDLINE und Google Scholar nach Studien, die zwischen Januar 1998 und Dezember 2017 veröffentlicht worden waren, und verwendeten dabei die Suchbegriffe Körperposition, Lungenfunktion, Lungenmechanik, Lungenvolumen, Positionsänderung, Positionierung, Körperhaltung, Lungenfunktionsprüfung, sitzend, stehend, Rückenlage, Ventilation und Ventilationsänderung (body position, lung function, lung mechanics, lung volume, position change, positioning, posture, pulmonary function testing, sitting, standing, supine, ventilation, and ventilatory change) in verschiedenen Kombinationen. Jede Suchbegriffkombination beinhaltete mindestens ein Schlüsselwort mit Bezug zur Lungenfunktion und mindestens ein Schlüsselwort mit Bezug zur Körperposition. Als Startpunkt wurde das Jahr 1998 gewählt, da in 1998 die wegweisende Studie von Meysman und Vincken veröffentlicht worden war [3]. Die beiden Wissenschaftlerinnen überprüften insgesamt 972 im Rahmen der Suche identifizierte Abstracts und riefen von 151 potenziell relevanten Artikeln die Volltextversion ab. Die vollständigen Texte wurden bewertet und kategorisiert und 108 Artikel, die die Einschlusskriterien nicht erfüllten, wurden ausgeschlossen (Abb. 1).

Artikel, die die folgenden Kriterien erfüllten, wurden eingeschlossen: (1) quasi-experimentelle Prä-post-Intervention; (2) 
Vergleich von mindestens 2 Körperpositionen, darunter mindestens Sitzen oder Stehen; (3) Zielkriterien umfassten die Beurteilung der Lungenfunktion anhand der forcierten Vitalkapazität (forced vital capacity, FVC), des forcierten exspiratorischen Volumens in 1 Sekunde $\left(\mathrm{FEV}_{1}\right), \mathrm{FEV}_{1} / \mathrm{FVC}$, der Vitalkapazität (VC), der funktionellen Residualkapazität (functional residual capacity, FRC), des maximalen exspiratorischen Drucks (maximal expiratory pressure, PEmax), des maximalen inspiratorischen Drucks (maximal inspiratory pressure, PImax), des exspiratorischen Spitzenflusses (peak expiratory flow, PEF), der totalen Lungenkapazität (total lung capacity, TLC), des Residualvolumens (RV) oder der Kohlenmonoxid-Diffusionskapazität der Lunge (diffusing capacity of the lungs for carbon monoxide, $\mathrm{D}_{\mathrm{LCO}}$ ); (4) Studienpopulation von nicht mechanisch beatmeten Probanden; (5) Teilnehmer $\geq 18$ Jahre; (6) Veröffentlichung auf Englisch. Studien, die andere Bewertungskriterien zur Beurteilung der Lungenfunktion verwendeten, sowie solche ohne statistische Vergleiche der Lungenfunktion in verschiedenen Körperpositionen, außerdem Studien, deren Teilnehmer jünger waren als 18 Jahre oder mechanisch beatmet wurden, und Veröffentlichungen von Kongress-Abstracts und systematische Übersichtsarbeiten wurden ausgeschlossen.

\section{Untersuchte Körperpositionen}

- Stehend - nicht unterstütztes, aktives Stehen

- Sitzend - Sitzen auf einem Stuhl oder in einem Rollstuhl mit der Rückenlehne in $90^{\circ}$-Position und Unterstützung aller Gliedmaßen

- Rückenlage - flach auf dem Rücken liegend

- Rechtsseitenlage (RSL) - ausgestreckt auf der rechten Seite liegend

- Linksseitenlage (LSL) - ausgestreckt auf der linken Seite liegend

\section{Zielkriterien und definierte Schwellenwerte für klinische}

\section{Relevanz}

- FVC, Änderung um $200 \mathrm{ml}$ oder $12 \%$ gegenüber den FVCAusgangswerten [4]

- $\mathrm{FEV}_{1}$, Änderung um $200 \mathrm{ml}$ oder $12 \%$ gegenüber den $\mathrm{FEV}_{1^{-}}$ Ausgangswerten [4]

- $\mathrm{FEV}_{1} / \mathrm{FVC}\left(\mathrm{FEV}_{1} / \mathrm{FVC}<0,7\right.$ ist definiert als obstruktive Erkrankung)

- $\mathrm{VC}$

- FRC, Änderung $>10 \%[5]$

- TLC, Änderung > 10\% [5]

- $\mathrm{RV}$

- PEmax, Änderung $\geq 24 \mathrm{~cm} \mathrm{H}_{2} \mathrm{O}$ [6-8]

- PImax, Änderung $\leq-13 \mathrm{~cm} \mathrm{H}_{2} \mathrm{O}[6-8]$

- PEF, Änderung $>10 \%$ oder $601 / \min [9,10]$

- $\mathrm{D}_{\mathrm{LCO}}, \ddot{A}$ derung $\geq 10 \%[11,12]$

Zwei erfahrene Pneumologen (N.A., A.R.) überprüften die eingeschlossenen Studien in Abstimmung und identifizierten statistisch signifikante und klinisch bedeutsame Unterschiede in der Lungenfunktion. Die Ergebnisse der in die Übersichtsarbeit ein- geschlossenen Artikel wurden von allen Autoren geprüft und nach Studienpopulation, untersuchten Körperpositionen und Zielkriterien klassifiziert. 4 Autoren (N.A., A.R., S.K., E.-L.M.) nahmen unabhängig voneinander die Datenextraktion aus den eingeschlossenen Studien vor und hielten bei etwaigen Fragen Rücksprache. Die Erstellung der Übersichtsarbeit erfolgte gemäß den PRISMA-Leitlinien [13].

Obwohl es sich streng genommen nicht um interventionelle Studien handelt, entschieden wir uns, sie als «Vorher-Nachher-Intervention» anzusehen, mit der Änderung der Körperhaltung/Körperposition als interessierendem Manöver. Zur Beurteilung des Evidenzgrades wurde die Klassifikation der Evidenz für therapeutische Interventionen der American Academy of Neurology herangezogen [14]. Die Bewertung des Voreingenommenheitsrisikos (Bias-Risiko) erfolgte mithilfe des Quality Assessment Tool for Before-After (Pre-Post) Studies with No Control Group des National Heart, Lung and Blood Institute (NHLBI) der US-amerikanischen National Institutes of Health (NIH) [15]. Dieses Instrument umfasst 12 Fragen zur Bewertung verschiedener Aspekte der Studienqualität. Mithilfe der Methode von Kunstler et al. [16] bewerteten 2 Autorinnen (E.-L.M., S.K.) unabhängig voneinander die einzelnen Studien mit Punkten. Etwaige Unterschiede wurden einvernehmlich und in Abstimmung mit einem dritten Autor (Y.Z.) geklärt. Das Bias-Risiko wurde als niedrig (Score 76$100 \%)$, mäßig (26-75\%) oder hoch (0-25\%) eingestuft.

\section{Ergebnisse}

\section{In die Übersichtsarbeit aufgenommene Studien}

Insgesamt erfüllten 43 Studien die Einschlusskriterien und wurden in die Übersichtsarbeit aufgenommen (Abb. 1). In allen Studien kam ein konsekutives, willkürliches oder freiwilliges Stichprobenverfahren zur Anwendung, um gesunde Probanden oder Patienten mit verschiedenen Erkrankungen zu rekrutieren. Alle Studien weisen einen Evidenzgrad der Klasse III auf.

Die Protokolle und Bias-Scores der verschiedenen Studien sind in Tabelle 1 und Tabelle S1 (siehe Zusatzmaterial) aufgeführt. Das Bias-Risiko wurde bei 41 Studien als mäßig eingestuft und bei 2 Studien als niedrig. Qualitätsprobleme waren hauptsächlich durch das zur Rekrutierung der Studienteilnehmer verwendete Stichprobenverfahren bedingt. In allen Studien wurde ein nicht randomisiertes Stichprobenverfahren verwendet. Einige Studien, in denen gesunde Probanden untersucht wurden, schlossen willkürlich ausgewählte Stichproben von jungen Teilnehmern, überwiegend Studenten, ein. Nur 7 von 43 Studien enthielten Angaben zu den Berechnungen des für die statistische Macht erforderlichen Stichprobenumfangs. Darüber hinaus wurden in einigen Studien keine klaren Angaben zu den Einzelheiten der Interventionsprotokolle gemacht (Tab. 1) und eine Verblindung in Hinblick auf die Körperposition des Patienten oder die Ergebnisse vorheriger Untersuchungen war aufgrund der Natur der Studienbewerter nicht möglich.

Die Studiencharakteristika, einschließlich der untersuchten Körperpositionen, Zielkriterien und Hauptergebnisse nach Studien- 
Tab. 1. Studienprotokolle

\begin{tabular}{|c|c|c|c|c|}
\hline Erstautor (Jahr) & Verfahren & $\begin{array}{l}\text { Randomisierung von Körperhaltung } \\
\text { und Test }\end{array}$ & $\begin{array}{l}\text { Adaptationsphase } \\
\text { an die Körper- } \\
\text { haltung vor der } \\
\text { Messung }\end{array}$ & $\begin{array}{l}\text { Bias- } \\
\text { risiko }\end{array}$ \\
\hline Antunes (2016) [45] & $\begin{array}{l}\text { Mini Wright }{ }^{\oplus} \text { (Clement Clarke International } \\
\text { Ltd. Edinburgh Way Harlow, Essex, UK) } \\
\text { tragbares Peak-Flow-Meter mit Einmal- } \\
\text { Mundstück }\end{array}$ & Zufällige Positionsreihenfolge & $1 \mathrm{~min}$ & Mäßig \\
\hline $\operatorname{Badr}(2002)[46]$ & $\begin{array}{l}\text { Druckmanometer, Vitalograph (Compact, } \\
\text { Vitalograph Ltd., Buckingham, UK) }\end{array}$ & $\begin{array}{l}\text { Zufällige Positionsreihenfolge } \\
\text { Zufällige Testreihenfolge (PEF und } \\
\text { PEmax); Einweisung der Probanden in die } \\
\text { Handhabung der Geräte, Übungsphase vor } \\
\text { dem Test }\end{array}$ & $5 \mathrm{~min}$ & Niedrig \\
\hline Baydur (2001) [35] & Spirometrie & Zufällige Positionsreihenfolge & k. A. & Mäßig \\
\hline Ben-Dov (2009) [17] & Spirometrie & k. A. & k. A. & Mäßig \\
\hline Benedik (2009) [52] & Heliumdilution & Erste Position immer sitzend & $5 \mathrm{~min}$ & Mäßig \\
\hline Ceridon (2011) [18] & $\begin{array}{l}\text { Spirometrie, } D_{\text {Lco gemessen mittels }} \\
\text { Rückatmung }\end{array}$ & k. A. & $\begin{array}{l}30 \text { min Rückenlage } \\
\text { vor dem Test; Zeit } \\
\text { vor der Messung } \\
\text { im Sitzen nicht } \\
\text { angegeben }\end{array}$ & Mäßig \\
\hline Chang (2005) [53] & $\begin{array}{l}\text { Spirometrie, FRC gemessen mittels } \\
\text { Heliumdilution }\end{array}$ & Erste Position immer Rückenlage & $5 \mathrm{~min}$ & Mäßig \\
\hline Costa (2015) [54] & $\begin{array}{l}\text { Mundverschlussdruck- } \\
\text { Messgerät }\end{array}$ & Zufällige Positionsreihenfolge & $10 \mathrm{~min}$ & Mäßig \\
\hline De (2012) [29] & Spirometrie & Erste Position immer sitzend & k. A. & Mäßig \\
\hline Elkins (2005) [47] & $\begin{array}{l}\text { Druckmessgerät, Spirometrie - } \\
\text { Massenflusssensor }\end{array}$ & $\begin{array}{l}\text { Zufällige Position und Testreihenfolge (PEF, } \\
\text { PEmax) } \\
\text { Einweisung der Probanden in die } \\
\text { Handhabung der Geräte, Übungsphase vor } \\
\text { dem Test }\end{array}$ & $5 \mathrm{~min}$ & Niedrig \\
\hline Faggiano (1998) [58] & $\begin{array}{l}\text { Einzelatemzugtechnik unter Verwendung } \\
\text { eines Medical Graphics PF/DX Moduls } \\
\text { (Medical Graphics St. Paul, Minn, USA) zur } \\
\text { Bestimmung der DLCO }\end{array}$ & Zufällige Positionsreihenfolge & $10 \mathrm{~min}$ & Mäßig \\
\hline Ganapathi (2015) [19] & $\begin{array}{l}\text { Digitale Spirometrie (BIOPAC System Inc. } \\
\text { Goleta, California, USA) }\end{array}$ & k. A. & k. A. & Mäßig \\
\hline Gianinis (2013) [48] & Tragbares Peak-Flow-Meter & Zufällige Positionsreihenfolge & k. A. & Mäßig \\
\hline $\operatorname{Kim}(2012)[36]$ & Spirometrie & k. A. & k. A. & Mäßig \\
\hline Linn (2000) [33] & Spirometrie & Zufällige Positionsreihenfolge & k. A. & Mäßig \\
\hline Manning (1999) [20] & $\begin{array}{l}\text { Spirometrie, Einzelatemzugtechnik zur } \\
\text { Bestimmung der } D_{\mathrm{LCO}}\end{array}$ & $\begin{array}{l}\text { Zwei Protokolle (Sitzung A u. B). Erste Position } \\
\text { zufällig festgelegt; danach abwechselnd bei } \\
\text { den nachfolgenden Probanden } \\
\text { Erste Position immer sitzend }\end{array}$ & $15 \mathrm{~min}$ & Mäßig \\
\hline McCoy (2010) [49] & Peak-Flow-Meter & $\begin{array}{l}\text { Zufällige Positionsreihenfolge } \\
\text { Einweisung der Probanden } \\
\text { in die Handhabung der Geräte, Übungsphase } \\
\text { vor dem Test }\end{array}$ & k. A. & Mäßig \\
\hline Melam (2014) [30] & $\begin{array}{l}\text { Spirometrie (Excel/PC-basierte } \\
\text { Lungenfunktionstests) }\end{array}$ & Zufällige Positionsreihenfolge & k. A. & Mäßig \\
\hline Meysman (1998) [3] & Spirometrie, Peak-Flow-Meter & Zufällige Positionsreihenfolge & $10 \mathrm{~min}$ & Mäßig \\
\hline Miccinilli (2016) [40] & Spirometrie & k. A. & k. A. & Mäßig \\
\hline $\begin{array}{l}\text { Mohammed (2017) } \\
\text { [31] }\end{array}$ & Spirometrie & $\begin{array}{l}\text { Reihenfolge immer stehend, sitzend, } \\
\text { Rückenlage, Seitenlage }\end{array}$ & k. A. & Mäßig \\
\hline Myint (2017) [42] & Spirometrie & Reihenfolge stehend, sitzend, Rückenlage & k. A. & Mäßig \\
\hline Naitoh (2014) [39] & $\begin{array}{l}\text { Spirometrie, Atem-Dynamometer } \\
\text { (Chest Co. Ltd) }\end{array}$ & Erste Position immer sitzend & k. A. & Mäßig \\
\hline Ogiwara (2002) [55] & $\begin{array}{l}\text { Vitalpower KH-101 } \\
\text { (Chest M.I. Inc., Japan) }\end{array}$ & Zufällige Positionsreihenfolge & $10 \mathrm{~min}$ & Mäßig \\
\hline
\end{tabular}


Tab. 1 (Fortsetzung)

\begin{tabular}{|c|c|c|c|c|}
\hline Erstautor (Jahr) & Verfahren & $\begin{array}{l}\text { Randomisierung von Körperhaltung } \\
\text { und Test }\end{array}$ & $\begin{array}{l}\text { Adaptationsphase } \\
\text { an die Körper- } \\
\text { haltung vor der } \\
\text { Messung }\end{array}$ & $\begin{array}{l}\text { Bias- } \\
\text { risiko }\end{array}$ \\
\hline Ottaviano (2016) [50] & Peak-Flow-Meter & Zufällige Positionsreihenfolge & k. A. & Mäßig \\
\hline Palermo (2005) [21] & $\begin{array}{l}\text { Spirometrie, } D_{\text {Lco gemessen mittels }} \\
\text { Einzelatemzugtechnik }\end{array}$ & Zufällige Positionsreihenfolge & $15 \mathrm{~min}$ & Mäßig \\
\hline Park (2010) [34] & Spirometrie & k. A. & k. A. & Mäßig \\
\hline Patel (2015) [22] & Spirometrie & Erste Position immer sitzend & k. A. & Mäßig \\
\hline $\begin{array}{l}\text { Peces-Barba (2004) } \\
\text { [56] }\end{array}$ & $\begin{array}{l}\text { Einzelatemzugtechnik, } \\
\text { Rückatmungstechnik } \\
\text { zur Bestimmung der } D_{\mathrm{LCO}}\end{array}$ & k. A. & $3-5 \min$ & Mäßig \\
\hline Poussel (2014) [38] & Spirometrie & Zufällige Positionsreihenfolge & k. A. & Mäßig \\
\hline Razi (2007) [32] & Spirometrie & $\begin{array}{l}\text { Abwechselnd sitzend } \\
\text { und stehend }\end{array}$ & $\begin{array}{l}\text { k. A.i } 15 \text { min } \\
\text { zwischen den } \\
\text { Positionen }\end{array}$ & Mäßig \\
\hline $\begin{array}{l}\text { Roychowdhury (2011) } \\
\text { [44] }\end{array}$ & Spirometrie & k. A. & $\begin{array}{l}\text { k. A. } \\
5 \text { min Ruhephase } \\
\text { zwischen den } \\
\text { Positionen }\end{array}$ & Mäßig \\
\hline Saxena (2006) [23] & Spirometrie & k. A. & k. A. & Mäßig \\
\hline Sebbane (2015) [41] & $\begin{array}{l}\text { Spirometrie, Mehrfachatemzug-Helium- } \\
\text { Dilutionsmethode }\end{array}$ & Erste Position immer sitzend & k. A. & Mäßig \\
\hline Stewart (2000) [24] & $\begin{array}{l}\text { Einzelatemzugtechnik zur Bestimmung der } \\
D_{\text {LCO }}\end{array}$ & k. A.; 72 h zwischen den Positionen & $15 \mathrm{~min}$ & Mäßig \\
\hline $\begin{array}{l}\text { Terson de Paleville } \\
\text { (2014) [37] }\end{array}$ & $\begin{array}{l}\text { Spirometrie, MP45-36-350 } \\
\text { Differentialdruckwandler Validyne } \\
\text { Engineering, (Northridge Ca, USA) }\end{array}$ & Erste Position immer sitzend & $30 \mathrm{~min}$ & Mäßig \\
\hline Terzano (2009) [57] & $\begin{array}{l}\text { Einzelatemzugtechnik zur Bestimmung der } \\
D_{\text {LCO }}\end{array}$ & Zufällige Positionsreihenfolge & $\begin{array}{l}\text { Mindestens } \\
15 \mathrm{~min}\end{array}$ & Mäßig \\
\hline Tsubaki (2009) [28] & $\begin{array}{l}\text { Micro RPM } 01 \text { (Micro Medical, UK), } \\
\text { Spirometrie }\end{array}$ & Zufällige Positionsreihenfolge & k. A. & Mäßig \\
\hline Varrato (2001) [25] & Spirometrie & k. A. & k. A. & Mäßig \\
\hline Vilke (2000) [26] & Spirometrie & Erste Position immer Rückenlage/Bauchlage & k. A. & Mäßig \\
\hline Wallace (2013) [51] & Peak-Flow-Meter & Zufällige Positionsreihenfolge & k. A. & Mäßig \\
\hline Watson (2005) [43] & $\begin{array}{l}\text { Mehrfachatemzug-Helium-Dilution, } \\
\text { Spirometrie }\end{array}$ & k. A. & k. A. & Mäßig \\
\hline Yap (2000) [27] & $\begin{array}{l}\text { Spirometrie, FRC gemessen mittels } \\
\text { Heliumdilution }\end{array}$ & Erste Position immer sitzend & $5 \mathrm{~min}$ & Mäßig \\
\hline
\end{tabular}

Die Bewertung des Verzerrungs (Bias-)risikos erfolgte mithilfe des Quality Assessment Tool for Before-After (Pre-Post) Studies with No Control Group [15, 16]. $\mathrm{D}_{\mathrm{Lco}}$ : Diffusing capacity of the lungs for carbon monoxide (Kohlenmonoxid-Diffusionskapazität der Lunge); FRC: Functional residual capacity (funktionelle Residualkapazität); k. A.: Keine Angaben, in der Studie nicht berichtet.

population sind in Tabelle 2 zusammengefasst. 29 von 43 Studien schlossen gesunde Probanden ein, 9 Studien schlossen Patienten mit Lungenerkrankungen ein, 4 Studien Patienten mit Herzerkrankungen, 7 Studien Patienten mit Rückenmarksverletzung, 3 Studien Patienten mit neuromuskulären Erkrankungen und 4 Studien schlossen Patienten mit Adipositas ein. In Tabelle S2 (siehe Zusatzmaterial) sind nur die statistisch signifikanten Ergebnisse für die einzelnen relevanten Zielkriteriumsvariablen nach Position für die jeweils untersuchten Populationen zusammengefasst.
FVC

Der Zusammenhang zwischen FVC und Körperposition bei gesunden Probanden wurde in 13 Studien untersucht [3, 17-28]. Es bestand ein klinisch bedeutsamer und statistisch signifikanter Anstieg der FVC-Werte im Sitzen versus Rückenlage [3, 18, 2227], im Sitzen versus RSL und LSL [3, 21], im Stehen versus Rückenlage [19, 23] und im Stehen versus RSL und LSL [19]. In einer kleineren Anzahl von Studien war keine Veränderung zwischen der stehenden und der sitzenden Position [19], zwischen Stehen und Rückenlage $[17,21,28]$ oder zwischen Sitzen und RSL oder LSL [21] zu beobachten und in einer Studie [22] kam es beim 
Wechsel vom Sitzen zum Stehen zu einem statistisch signifikanten - jedoch nicht klinisch bedeutsamen - Rückgang der FVCWerte. Das heißt, in den meisten Studien war die aufrechte Position mit höheren FVC-Werten assoziiert.

Vier Studien schlossen Patienten mit Lungenerkrankungen ein [29-32]. In einer Studie stieg der FVC-Wert bei Asthma-Patienten beim Wechsel von der Rückenlage zum Stehen signifikant an [30], doch war kein signifikanter Unterschied zwischen der stehenden und sitzenden Position oder zwischen Sitzen und Rückenlage, RSL oder LSL feststellbar. Eine weitere Studie berichtete einen statistisch signifikanten und klinisch bedeutsamen Anstieg der FVC im Stehen versus Sitzen, Rückenlage, RSL und LSL und im Sitzen versus Rückenlage, RSL und LSL [31]. Bei adipösen Asthmatikern [32] und bei Patienten mit chronisch obstruktiver Lungenerkrankung (chronic obstructive pulmonary disease, COPD) [29] zeigte sich kein Unterschied in den FVC-Werten zwischen Stehen und Sitzen.

Drei Studien schlossen Patienten mit kongestiver Herzinsuffizienz (congestive heart failure, CHF) ein [18, 21, 27]. In einer Studie fiel die FVC im Sitzen um $200 \mathrm{ml}$ höher aus als in RSL und LSL [21], und in den anderen beiden Studien war sie im Sitzen 350-400 $\mathrm{ml}$ höher als in Rückenlage, ein Ergebnis, das klinisch bedeutsam war [18, 27].

Sechs Studien schlossen Patienten mit Rückenmarksverletzung ein [17, 33-37]. Der Einfluss der Körperposition auf die FVC hängt von der Höhe und dem Ausmaß der Verletzung ab. Bei denjenigen mit zervikaler Rückenmarksverletzung fielen die FVCWerte in Rückenlage höher aus als im Sitzen [17, 33, 34]. Andere Studien [35-37] ergaben keine signifikanten Unterschiede in den FVC-Werten bei Patienten mit Rückenmarksverletzung in einer gepoolten Gruppe aller Verletzungshöhen für diese Positionen. Allerdings zeigten Patienten mit zervikaler Rückenmarksverletzung sowie Patienten mit thorakaler Verletzung in einer Studie [36] in Rückenlage höhere FVC-Werte als im Sitzen, wohingegen die FVC-Werte bei Patienten mit thorakaler oder lumbaler Verletzung im Sitzen höher waren [37]. Die Unterschiede erreichten nicht immer statistische Signifikanz. Es ist jedoch zu beachten, dass bei diesen schwer beeinträchtigten Patienten mit Rückenmarksverletzung selbst eine kleine Änderung der FVC wahrscheinlich klinisch bedeutsam ist.

Drei Studien beurteilten Patienten mit neuromuskulären Erkrankungen $[25,34,38]$. Bei Patienten mit myotoner Dystrophie und solchen mit amyotropher Lateralsklerose (ALS) war ein klinisch bedeutsamer und statistisch signifikanter Rückgang der FVCWerte beim Wechsel vom Sitzen in die Rückenlage zu beobachten [25, 34, 38]. Bei Patienten mit Adipositas (mittlerer Body Mass Index (BMI): 36,7) wurde kein signifikanter Unterschied zwischen Stehen und Sitzen festgestellt [32].

\section{FEV}

Berichten zufolge waren die $\mathrm{FEV}_{1}$-Werte bei gesunden Probanden im Sitzen höher als in Rückenlage [3, 18, 22, 23, 26, 27, 39], im Sitzen höher als in RSL und LSL [3, 19, 20], im Stehen höher als im Sitzen [23] und im Stehen höher als im Sitzen, Rückenlage, RSL und LSL [19]. In anderen Studien [21, 24, 28, 40] fand sich jedoch kein signifikanter Unterschied in den $\mathrm{FEV}_{1}$-Werten zwischen Sitzen und Rückenlage, RSL und LSL. Eine Studie [22] berichtete einen statistisch signifikanten - allerdings klinisch nicht bedeutsamen - Rückgang der $\mathrm{FEV}_{1}$-Werte um $120 \mathrm{ml}$ beim Wechsel vom Sitzen zum Stehen.

Bei Asthma-Patienten lagen die $\mathrm{FEV}_{1}$-Werte im Stehen höher als im Sitzen, und die Unterschiede fielen statistisch signifikant und klinisch bedeutsam aus. Allerdings war kein signifikanter Unterschied im Sitzen versus Rückenlage, RSL und LSL zu beobachten [30]. Eine andere Studie mit Asthmapatienten berichtete über höhere $\mathrm{FEV}_{1}$-Werte im Stehen versus Sitzen, Rückenlage, RSL und LSL sowie im Sitzen versus Rückenlage, RSL und LSL [31]. Bei adipösen Asthmatikern und COPD-Patienten bestand kein signifikanter Unterschied im $\mathrm{FEV}_{1}$ zwischen Stehen und Sitzen [29, 32].

Patienten mit CHF zeigten in einer Studie einen statistisch signifikanten und klinisch bedeutsamen Anstieg der FEV1-Werte im Sitzen versus RSL und LSL, jedoch keinen Unterschied zwischen Sitzen und Rückenlage [21], wohingegen 2 andere Studien höhere $\mathrm{FEV}_{1}$-Werte im Sitzen versus Rückenlage berichteten [18, 27].

Kürzlich veröffentlichten Berichten zufolge steigen die $\mathrm{FEV}_{1^{-}}$ Werte bei Patienten mit Rückenmarksverletzung beim Wechsel vom Sitzen zur Rückenlage an [40], während der Einfluss der Körperposition auf die $\mathrm{FEV}_{1}$-Werte von Patienten mit Rückenmarksverletzung nach den Ergebnissen anderer Studien von der Höhe und dem Ausmaß der Verletzung abhängt. In einer Studie hatte der Wechsel vom Sitzen in die Rückenlage bei allen Patienten mit Rückenmarksverletzung keinen signifikanten Einfluss auf die $\mathrm{FEV}_{1}$-Werte [35], doch zeigten Patienten mit zervikalen Verletzungen tendenziell höhere $\mathrm{FEV}_{1}$-Werte in Rückenlage als im Sitzen, wohingegen die $\mathrm{FEV}_{1}$-Werte bei Patienten mit thorakalen Verletzungen tendenziell im Sitzen höher ausfielen. Ebenso war in einer anderen Studie [36] bei Patienten mit lumbaler Verletzung ein $\mathrm{FEV}_{1}$-Anstieg im Sitzen versus Rückenlage zu beobachten, während Patienten mit Verletzungen der Halswirbelsäule oder thorakalen Verletzungen in Rückenlage höhere $\mathrm{FEV}_{1}$-Werte aufwiesen. Zwar erreichten die Unterschiede zwischen den Positionen keine statistische Signifikanz, doch war der Einfluss der Verletzungshöhe statistisch signifikant und klinisch bedeutsam. In einer anderen Studie [33] waren die $\mathrm{FEV}_{1}$-Werte bei Patienten mit kompletter Tetraplegie in Rückenlage höher als im Sitzen, wohingegen bei Patienten mit inkompletter Lähmung kein signifikanter Unterschied zwischen den Positionen nachweisbar war. Eine andere Forschungsgruppe [37] berichtete keine signifikante Änderung der $\mathrm{FEV}_{1}$-Werte zwischen der sitzenden Position und der Rückenlage in einer gepoolten Gruppe von Patienten mit Rückenmarksverletzung; in der Subgruppe von Patienten mit inkompletter motorischer Lähmung und solchen mit inkompletter thorakaler Lähmung war dagegen ein Rückgang in Rückenlage zu beobachten.

Bei Patienten mit myotoner Dystrophie gingen die $\mathrm{FEV}_{1}$-Werte beim Wechsel vom Sitzen in die Rückenlage zurück [38]. Adipöse Patienten wiesen sowohl vor als auch nach einer bariatrischen 
Tab. 2 Zusammenfassung der Studiencharakteristika nach Studienpopulation

\begin{tabular}{|c|c|c|c|c|c|c|}
\hline Erstautor (Jahr) & $\begin{array}{l}\text { Teilnehmer- } \\
\text { zahl }\end{array}$ & $\begin{array}{l}\text { Alter } \\
\text { (Jahre) }\end{array}$ & $\begin{array}{l}\text { Patienten- } \\
\text { population }\end{array}$ & Positionen & $\begin{array}{l}\text { Lungenfunktions- } \\
\text { prüfung }\end{array}$ & Hauptergebnisse \\
\hline $\begin{array}{l}\text { Meysman (1998) } \\
\text { [3] }\end{array}$ & 31 & $25,6 \pm 3,8$ & Gesunde & $\begin{array}{l}\text { Sitzend } \\
\text { Rückenlage } \\
\text { RSL } \\
\text { LSL }\end{array}$ & $\begin{array}{l}\text { PEF, FVC, FEV } \\
\text { PImax, PEmax }\end{array}$ & $\begin{array}{l}\text { FVC, FEV } 1 \text {, PEF: Sitzend > Rückenlage } \\
\text { LSL, RSL, PImax: Sitzend > Rückenlage } \\
\text { PEmax: } p>0,05 \text { zwischen den Positionen }\end{array}$ \\
\hline $\begin{array}{l}\text { Manning (1999) } \\
\text { [20] }\end{array}$ & 19 & $62,8 \pm 6,8$ & $\begin{array}{l}\text { Gesunde ältere } \\
\text { Erwachsene }\end{array}$ & $\begin{array}{l}\text { Sitzend } \\
\text { LSL } \\
\text { RSL }\end{array}$ & $\mathrm{FVC}, \mathrm{FEV}{ }_{1} \mathrm{D}_{\mathrm{LCO}} / \mathrm{VA}$ & $\begin{array}{l}\text { FVC, FEV } \text { : Sitzend }>\text { RSL u. LSL } \\
D_{L C O} / N A: p>0,05 \text { zwischen den Positionen }\end{array}$ \\
\hline Vilke (2000) [26] & 20 & $\begin{array}{l}\text { Spanne: } \\
18-50\end{array}$ & $\begin{array}{l}\text { Gesunde } \\
\text { Männer }\end{array}$ & $\begin{array}{l}\text { Sitzend } \\
\text { Rückenlage }\end{array}$ & FVC, FEV & FVC, FEV 1 : Sitzend > Rückenlage \\
\hline $\begin{array}{l}\text { Stewart (2000) } \\
{[24]}\end{array}$ & 10 & $22,3 \pm 2,4$ & $\begin{array}{l}\text { Gesunde } \\
\text { Männer }\end{array}$ & $\begin{array}{l}\text { Sitzend } \\
\text { Rückenlage }\end{array}$ & $\begin{array}{l}\text { FVC, VC, FEV } \\
\mathrm{FEV}_{1} / \mathrm{FVC}, \mathrm{PEF} \\
\mathrm{D}_{\mathrm{LCO}}\end{array}$ & $\begin{array}{l}\text { FVC, PEF: Sitzend }>\text { Rückenlage } \\
D_{\text {LCO: }} \text { Sitzend }<\text { Rückenlage } \\
\text { FEV }_{1}, V C, F E V_{1} / F V C: p>0,05 \text { zwischen den } \\
\text { Positionen }\end{array}$ \\
\hline Yap (2000) [27] & 10 & $\begin{array}{l}62,2 \pm 1,2 \\
M W \pm S E\end{array}$ & Gesunde & $\begin{array}{l}\text { Sitzend } \\
\text { Rückenlage }\end{array}$ & $\begin{array}{l}\text { FVC, FEV }, \mathrm{FEV}_{1} / \\
\text { FVC, FRC }\end{array}$ & $\begin{array}{l}\text { FVC, FEV } 1, F R C: \text { Sitzend }>\text { Rückenlage } \\
\text { FEV }_{1} / F V C: p>0,05 \text { zwischen den Positionen }\end{array}$ \\
\hline $\begin{array}{l}\text { Varrato (2001) } \\
\text { [25] }\end{array}$ & 15 & MW: 41 & Gesunde & $\begin{array}{l}\text { Sitzend } \\
\text { Rückenlage }\end{array}$ & FVC & FVC: Sitzend > Rückenlage \\
\hline $\operatorname{Badr}(2002)[46]$ & 25 & $34,0 \pm 14,9$ & Gesunde & $\begin{array}{l}\text { Stehend } \\
\text { Sitzend } \\
\text { Rückenlage } \\
\text { RSL }\end{array}$ & $\begin{array}{l}\text { PEmax, } \\
\text { PEF }\end{array}$ & $\begin{array}{l}\text { PEmax: Stehend }>\text { andere Positionen } \\
\text { PEmax: Sitzend }>\text { Rückenlage u. RSL } \\
\text { PEF: Stehend }>\text { andere Positionen }\end{array}$ \\
\hline $\begin{array}{l}\text { Ogiwara (2002) } \\
\text { [55] }\end{array}$ & 20 & $\begin{array}{l}\text { MW: } \\
22,8 \pm 2,1 \\
\text { Spanne: } \\
21-28\end{array}$ & Gesunde & $\begin{array}{l}\text { Sitzend } \\
\text { Rückenlage } \\
\text { RSL } \\
\text { LSL }\end{array}$ & $\begin{array}{l}\text { PEmax, } \\
\text { PImax }\end{array}$ & $\begin{array}{l}\text { PEmax, PImax: } p>0,05 \text { zwischen den } \\
\text { Positionen }\end{array}$ \\
\hline $\begin{array}{l}\text { Peces-Barba } \\
(2004)[56]\end{array}$ & 14 & $37,5 \pm 11,5$ & Gesunde & $\begin{array}{l}\text { Sitzend } \\
\text { Rückenlage }\end{array}$ & $\mathrm{D}_{\mathrm{LCO}}$ & D LCO: Sitzend < Rückenlage \\
\hline $\begin{array}{l}\text { Chang (2005) } \\
\text { [53] }\end{array}$ & 20 & $28,3 \pm 4,8$ & $\begin{array}{l}\text { Gesunde } \\
\text { Männer }\end{array}$ & $\begin{array}{l}\text { Stehend } \\
\text { Rückenlage }\end{array}$ & FRC & FRC: Stehend > Rückenlage \\
\hline $\begin{array}{l}\text { Palermo (2005) } \\
\text { [21] }\end{array}$ & 14 & $61 \pm 8$ & Gesunde & $\begin{array}{l}\text { Sitzend } \\
\text { Rückenlage } \\
\text { LSL } \\
\text { RSL }\end{array}$ & $\begin{array}{l}\mathrm{FEV}_{1}, \mathrm{FVC}, \mathrm{VC} \\
\mathrm{D}_{\mathrm{LCO}}\end{array}$ & $\begin{array}{l}\mathrm{FEV}_{1}, \mathrm{FVC}, \mathrm{VC}: p>0,05 \text { zwischen den Positionen } \\
\mathrm{D}_{\mathrm{LCO}} \text { : Sitzend }>\text { LSL u. RSL }\end{array}$ \\
\hline $\begin{array}{l}\text { Watson (2005) } \\
{[43]}\end{array}$ & 5 & MW: 57 & Gesunde & $\begin{array}{l}\text { Sitzend } \\
\text { Rückenlage }\end{array}$ & $\begin{array}{l}\text { TLC, VC, } \\
\text { RV, FRC }\end{array}$ & $\begin{array}{l}\text { TLC, VC, RV: } p>0,05 \text { zwischen den Positionen } \\
\text { FRC: Sitzend > Rückenlage }\end{array}$ \\
\hline $\begin{array}{l}\text { Saxena (2006) } \\
\text { [23] }\end{array}$ & 80 & $\begin{array}{l}\text { Männer: } \\
21,3 \pm 1,5 \\
\text { Frauen: } \\
19,6 \pm 1,3 \\
\end{array}$ & Gesunde & $\begin{array}{l}\text { Stehend } \\
\text { Sitzend } \\
\text { Rückenlage }\end{array}$ & $\begin{array}{l}\mathrm{FEV}_{1}, \mathrm{FVC}, \mathrm{FEV}_{1} / \\
\text { FVC, PEF }\end{array}$ & $\begin{array}{l}\mathrm{FEV}_{1}, \mathrm{FVC}, \text { PEF: Stehend }>\text { Rückenlage } \\
\mathrm{FEV}_{1} / \mathrm{FVC}: \text { Sitzend }>\text { Rückenlage }\end{array}$ \\
\hline $\begin{array}{l}\text { Ben-Dov (2009) } \\
{[17]}\end{array}$ & 7 & $44 \pm 10$ & Gesunde & $\begin{array}{l}\text { Sitzend } \\
\text { Rückenlage }\end{array}$ & FVC & FVC: $p>0,05$ zwischen den Positionen \\
\hline $\begin{array}{l}\text { Terzano (2009) } \\
\text { [57] }\end{array}$ & 10 & $59,0 \pm 9,3$ & Gesunde & $\begin{array}{l}\text { Stehend } \\
\text { Sitzend } \\
\text { Rückenlage }\end{array}$ & $\mathrm{D}_{\mathrm{LCO}}$ & $\mathrm{D}_{\mathrm{LCO}}: p>0,05$ zwischen den Positionen \\
\hline $\begin{array}{l}\text { Tsubaki (2009) } \\
\text { [28] }\end{array}$ & 15 & $22,7 \pm 2,3$ & $\begin{array}{l}\text { Gesunde } \\
\text { Frauen }\end{array}$ & $\begin{array}{l}\text { Sitzend } \\
\text { Rückenlage }\end{array}$ & $\begin{array}{l}\mathrm{FVC}, \mathrm{FEV}_{1}, \mathrm{FEV}_{1} / \\
\text { FVC, VC, PImax, } \\
\text { PEmax }\end{array}$ & $\begin{array}{l}\text { FEV } 1 \text { /FVC: Sitzend }>\text { Rückenlage } \\
\text { FVC, FEV } 1, \text { VC, PImax, PEmax: } p>0,05 \text { zwischen } \\
\text { den Positionen }\end{array}$ \\
\hline $\begin{array}{l}\text { McCoy }(2010) \\
{[49]}\end{array}$ & 182 & $\begin{array}{l}23,5 \pm 2,5 \\
\text { (Gesunde } \\
\text { und } \\
\text { Asthmatiker) }\end{array}$ & Gesunde & $\begin{array}{l}\text { Stehend } \\
\text { Sitzend }\end{array}$ & PEF & PEF: $p>0,05$ zwischen den Positionen \\
\hline $\begin{array}{l}\text { Ceridon (2011) } \\
\text { [18] }\end{array}$ & 12 & $63 \pm 9$ & Gesunde & $\begin{array}{l}\text { Sitzend } \\
\text { Rückenlage }\end{array}$ & $\begin{array}{l}\mathrm{FVC}_{1} \mathrm{FEV}_{1}, \mathrm{FEV}_{1} / \\
\mathrm{FVC}, \mathrm{D}_{\mathrm{LCO}}\end{array}$ & $\begin{array}{l}\text { FVC, FEV } 1, D_{L C O}: \text { Sitzend }>\text { Rückenlage } \\
\text { FEV }_{1} / F_{V C}: p>0,05 \text { zwischen den Positionen }\end{array}$ \\
\hline $\begin{array}{l}\text { Roychowdhury } \\
\text { (2011) [44] }\end{array}$ & 100 & $\begin{array}{l}\text { Spanne: } \\
19-22\end{array}$ & Gesunde & $\begin{array}{l}\text { Sitzend } \\
\text { Rückenlage }\end{array}$ & VC & $\begin{array}{l}\text { VC: Rückenlage > Sitzend bei Frauen } \\
\text { VC: } p>0,05 \text { zwischen den Positionen bei } \\
\text { Männern }\end{array}$ \\
\hline
\end{tabular}


Tab. 2 (Fortsetzung)

\begin{tabular}{|c|c|c|c|c|c|c|}
\hline Erstautor (Jahr) & $\begin{array}{l}\text { Teilnehmer- } \\
\text { zahl }\end{array}$ & $\begin{array}{l}\text { Alter } \\
\text { (Jahre) }\end{array}$ & $\begin{array}{l}\text { Patienten- } \\
\text { population }\end{array}$ & Positionen & $\begin{array}{l}\text { Lungenfunktions- } \\
\text { prüfung }\end{array}$ & Hauptergebnisse \\
\hline $\begin{array}{l}\text { Gianinis (2013) } \\
\text { [48] }\end{array}$ & 30 & $22,2 \pm 2,4$ & Gesunde & $\begin{array}{l}\text { Sitzend } \\
\text { Rückenlage } \\
\text { RSL } \\
\text { LSL }\end{array}$ & PEF & PEF: Sitzend > Rückenlage u. RSL \\
\hline $\begin{array}{l}\text { Wallace (2013) } \\
{[51]}\end{array}$ & 94 & $23,9 \pm 3,7$ & Gesunde & $\begin{array}{l}\text { Stehend } \\
\text { Sitzend }\end{array}$ & PEF & PEF: Stehend $>$ Sitzend \\
\hline $\begin{array}{l}\text { Naitoh (2014) } \\
\text { [39] }\end{array}$ & 20 & $28 \pm 1,4$ & Gesunde & $\begin{array}{l}\text { Sitzend } \\
\text { Rückenlage }\end{array}$ & $\begin{array}{l}\text { FEV } 1, \text { VC PEmax, } \\
\text { PImax }\end{array}$ & $\begin{array}{l}\text { FEV } 1 \text {, VC: Sitzend }>\text { Rückenlage } \\
\text { PEmax, PImax: } p>0,05 \text { zwischen den } \\
\text { Positionen }\end{array}$ \\
\hline Costa (2015) [54] & 63 & $19,7 \pm 1,5$ & Gesunde & $\begin{array}{l}\text { Sitzend } \\
\text { Rückenlage }\end{array}$ & $\begin{array}{l}\text { PImax, } \\
\text { PEmax }\end{array}$ & PImax, PEmax: Sitzend > Rückenlage \\
\hline $\begin{array}{l}\text { Ganapathi (2015) } \\
\text { [19] }\end{array}$ & 20 & $\begin{array}{l}\text { Spanne: } \\
18-25\end{array}$ & Gesunde & $\begin{array}{l}\text { Stehend } \\
\text { Sitzend } \\
\text { Rückenlage } \\
\text { RSL } \\
\text { LSL }\end{array}$ & $\begin{array}{l}\text { FVC, } \mathrm{FEV}_{1}, \mathrm{FEV}_{1} / \\
\text { FVC }\end{array}$ & $\begin{array}{l}\text { FVC, FEV } 1, F^{\prime} V_{1} / \text { FVC: } \text { Stehend > Rückenlage, } \\
\text { RSL u. LSL FEV } 1 \text { : Stehend > Sitzend; Sitzend > } \\
\text { RSL; FEV } / \text { FVC: Sitzend > LSL }\end{array}$ \\
\hline Patel (2015) [22] & 45 & $\begin{array}{l}\text { Median: 21; } \\
\text { Spanne: } \\
\text { 19-23 }\end{array}$ & Gesunde & $\begin{array}{l}\text { Stehend } \\
\text { Sitzend } \\
\text { Rückenlage }\end{array}$ & FVC, FEV ${ }_{1}$, PEF & $\begin{array}{l}\text { FVC, FEV } 1, \text { PEF: Sitzend > Stehend FVC, FEV } \\
\text { PEF: Sitzend }>\text { Rückenlage }\end{array}$ \\
\hline $\begin{array}{l}\text { Antunes (2016) } \\
{[45]}\end{array}$ & 30 & $22,7 \pm 2,4$ & Gesunde & $\begin{array}{l}\text { Sitzend } \\
\text { Rückenlage }\end{array}$ & PEF & PEF: Sitzend > Rückenlage \\
\hline $\begin{array}{l}\text { Miccinilli (2016) } \\
{[40]}\end{array}$ & 20 & $33,6 \pm 10,5$ & Gesunde & $\begin{array}{l}\text { Sitzend } \\
\text { Rückenlage }\end{array}$ & VC, FEV & VC, FEV $1: p>0,05$ zwischen den Positionen \\
\hline $\begin{array}{l}\text { Ottaviano (2016) } \\
\text { [50] }\end{array}$ & 76 & $40 \pm 16$ & Gesunde & $\begin{array}{l}\text { Stehend } \\
\text { Sitzend }\end{array}$ & PEF & PEF: Stehend > Sitzend \\
\hline Myint (2017) [42] & 15 & $22,6 \pm 2,0$ & Gesunde & $\begin{array}{l}\text { Stehend } \\
\text { Sitzend } \\
\text { Rückenlage }\end{array}$ & $\mathrm{FEV}_{1} / \mathrm{FVC}$ & $\mathrm{FEV}_{1} / \mathrm{FVC}: p>0,05$ zwischen den Positionen \\
\hline Badr (2002) [46] & 11 & $66,8 \pm 12,6$ & $\begin{array}{l}\text { Chronische } \\
\text { Atemflussein- } \\
\text { schränkung }\end{array}$ & $\begin{array}{l}\text { Stehend } \\
\text { Sitzend } \\
\text { Rückenlage } \\
\text { RSL }\end{array}$ & $\begin{array}{l}\text { PEmax, } \\
\text { PEF }\end{array}$ & $\begin{array}{l}\text { PEmax: Stehend > Rückenlage u. RSL, PEmax: } \\
\text { Sitzend > Rückenlage u. RSL } \\
\text { PEF: Stehend > Sitzend, Rückenlage, RSL }\end{array}$ \\
\hline Elkins (2005) [47] & 20 & $29 \pm 8$ & $\begin{array}{l}\text { Erwachsene } \\
\text { mit zystischer } \\
\text { Fibrose }\end{array}$ & $\begin{array}{l}\text { Stehend } \\
\text { Sitzend } \\
\text { Rückenlage } \\
\text { RSL }\end{array}$ & $\begin{array}{l}\text { PEmax, } \\
\text { PEF }\end{array}$ & $\begin{array}{l}\text { PEmax: Stehend u. Sitzend }>\text { RSL } \\
\text { PEF: Stehend > Rückenlage u. RSL }\end{array}$ \\
\hline Razi (2007) [32] & 49 & $42,6 \pm 11,8$ & $\begin{array}{l}\text { Adipositas, } \\
\text { Asthma } \\
\text { (Mittlerer BMI: } \\
36 \pm 5 \text { ) }\end{array}$ & $\begin{array}{l}\text { Stehend } \\
\text { Sitzend }\end{array}$ & $\begin{array}{l}\mathrm{FVC}_{1} \mathrm{FEV}_{1}, \mathrm{FEV}_{1} / \\
\text { FVC }\end{array}$ & $\begin{array}{l}\mathrm{FVC}_{1} \mathrm{FEV}_{1}, \mathrm{FEV}_{1} / \mathrm{FVC}: p>0,05 \text { zwischen den } \\
\text { Positionen }\end{array}$ \\
\hline $\begin{array}{l}\text { Terzano (2009) } \\
{[57]}\end{array}$ & $\begin{array}{l}30 \\
\text { Leicht: } 10 \\
\text { Mäßig bis } \\
\text { schwer: } 10 \\
\text { Sehr schwer: } \\
10\end{array}$ & $\begin{array}{l}\text { Leicht: } \\
57,3 \pm 8,6 \\
\text { Mäßig bis } \\
\text { schwer: } \\
59,8 \pm 9,1 \\
\text { Sehr schwer: } \\
63,7 \pm 5,5\end{array}$ & COPD & $\begin{array}{l}\text { Stehend } \\
\text { Sitzend } \\
\text { Rückenlage }\end{array}$ & $\mathrm{D}_{\mathrm{LCO}}$ & $\mathrm{D}_{\mathrm{LCO}}: p>0,05$ zwischen den Positionen \\
\hline $\begin{array}{l}\text { McCoy }(2010) \\
{[49]}\end{array}$ & 29 & $\begin{array}{l}23,5 \pm 2,5 \\
\text { (Gesunde } \\
\text { und } \\
\text { Asthmatiker) }\end{array}$ & Asthma & $\begin{array}{l}\text { Stehend } \\
\text { Sitzend }\end{array}$ & PEF & PEF: $p>0,05$ zwischen den Positionen \\
\hline De (2012) [29] & 75 & $61,2 \pm 9,2$ & COPD & $\begin{array}{l}\text { Stehend } \\
\text { Sitzend }\end{array}$ & FVC, FEV $_{1}$ & FVC, $\mathrm{FEV}_{1}: p>0,05$ zwischen den Positionen \\
\hline $\begin{array}{l}\text { Melam (2014) } \\
{[30]}\end{array}$ & 30 & $34,3 \pm 3,7$ & Asthma & $\begin{array}{l}\text { Stehend } \\
\text { Sitzend } \\
\text { Rückenlage } \\
\text { RSL } \\
\text { LSL }\end{array}$ & FVC, FEV $_{1}$ & FVC, FEV 1 : Stehend > Rückenlage \\
\hline
\end{tabular}


Tab. 2 (Fortsetzung)

\begin{tabular}{|c|c|c|c|c|c|c|}
\hline Erstautor (Jahr) & $\begin{array}{l}\text { Teilnehmer- } \\
\text { zahl }\end{array}$ & $\begin{array}{l}\text { Alter } \\
\text { (Jahre) }\end{array}$ & $\begin{array}{l}\text { Patienten- } \\
\text { population }\end{array}$ & Positionen & $\begin{array}{l}\text { Lungenfunktions- } \\
\text { prüfung }\end{array}$ & Hauptergebnisse \\
\hline $\begin{array}{l}\text { Mohammed } \\
(2017)[31]\end{array}$ & 20 & $39,2 \pm 8,0$ & Asthma & $\begin{array}{l}\text { Stehend } \\
\text { Sitzend } \\
\text { Rückenlage } \\
\text { RSL } \\
\text { LSL }\end{array}$ & FVC, FEV 1, PEF & $\begin{array}{l}\text { FVC, FEV } 1, \text { PEF: Stehend }>\text { Rückenlage, RSL, } \\
\text { LSL } \\
\text { FVC, FEV } 1 \text { : Stehend }>\text { Sitzend }\end{array}$ \\
\hline Myint (2017) [42] & 15 & $22,3 \pm 2,0$ & Asthma & $\begin{array}{l}\text { Stehend } \\
\text { Sitzend } \\
\text { Rückenlage }\end{array}$ & $\mathrm{FEV}_{1} / \mathrm{FVC}$ & $\mathrm{FEV}_{1} / \mathrm{FVC}: p>0,05$ zwischen den Positionen \\
\hline $\begin{array}{l}\text { Faggiano (1998) } \\
\text { [58] }\end{array}$ & 32 & $59 \pm 10$ & CHF, Männer & $\begin{array}{l}\text { Sitzend } \\
\text { Rückenlage }\end{array}$ & $\mathrm{D}_{\mathrm{LCO}}$ & $\begin{array}{l}D_{L C O}: p>0,05 \text { zwischen den Positionen } \\
\downarrow D_{L C O} \text { im Sitzen bei einer Subgruppe von } \\
\text { Patienten mit einer Verringerung des mittleren } \\
\text { pulmonal-arteriellen Drucks in dieser Position } \\
\uparrow D_{L C O} \text { im Sitzen bei einer Subgruppe von } \\
\text { Patienten mit einem Anstieg des mittleren } \\
\text { pulmonal-arteriellen Drucks in dieser Position }\end{array}$ \\
\hline Yap (2000) [27] & 10 & $\begin{array}{l}61,4 \pm 2,0 \\
M W \pm S E\end{array}$ & $\mathrm{CHF}$ & $\begin{array}{l}\text { Sitzend } \\
\text { Rückenlage }\end{array}$ & $\begin{array}{l}\mathrm{FEV}_{1}, \mathrm{FVC}, \mathrm{FEV} V_{1} / \\
\text { FVC, VC, FRC }\end{array}$ & $\begin{array}{l}\text { FVC, FEV }, \text { VC: Sitzend }>\text { Rückenlage FEV } 1 / F V C \text {, } \\
\text { FRC: } p>0,05 \text { zwischen den Positionen }\end{array}$ \\
\hline $\begin{array}{l}\text { Palermo (2005) } \\
\text { [21] }\end{array}$ & 14 & $62 \pm 8$ & $\mathrm{CHF}$ & $\begin{array}{l}\text { Sitzend } \\
\text { Rückenlage } \\
\text { LSL } \\
\text { RSL }\end{array}$ & $\mathrm{FEV}_{1}, \mathrm{FVC}, \mathrm{VC}, \mathrm{D}_{\mathrm{LCO}}$ & $\begin{array}{l}\text { FEV } 1 \text {, FVC: Sitzend }>\text { RSL u. LSL } \\
D_{L C O}: \text { Sitzend }>\text { RSL u. LSL } \\
\text { VC: } p>0,05 \text { zwischen den Positionen }\end{array}$ \\
\hline $\begin{array}{l}\text { Ceridon (2011) } \\
\text { [18] }\end{array}$ & 24 & $65 \pm 8$ & $\mathrm{CHF}$ & $\begin{array}{l}\text { Sitzend } \\
\text { Rückenlage }\end{array}$ & $\begin{array}{l}\mathrm{FEV}_{1}, \mathrm{FVC}_{1}, \mathrm{FEV}_{1} / \\
\text { FVC, } \mathrm{D}_{\mathrm{LCO}}\end{array}$ & $\begin{array}{l}\text { FEV }_{1}, \text { FVC: Sitzend }>\text { Rückenlage; FEV } / \text { FVC: } \\
p>0,05 \text { zwischen den Positionen } \\
D_{\text {LCO }}: p>0,05 \text { zwischen den Positionen }\end{array}$ \\
\hline Linn (2000) [33] & $\begin{array}{l}222 \\
\text { Tetraplegie: } 98 \\
\text { Paraplegie: } \\
124\end{array}$ & $40 \pm 11$ & $\mathrm{SCl}$ & $\begin{array}{l}\text { Sitzend } \\
\text { Rückenlage }\end{array}$ & FVC, FEV 1, PEF & $\begin{array}{l}\text { FVC, FEV } 1, \text { PEF: Sitzend }< \\
\text { Rückenlage bei kompletter Tetraplegie } \\
\text { FVC, FEV } 1, \text { PEF: } p>0,05 \text { zwischen den } \\
\text { Positionen bei Paraplegie }\end{array}$ \\
\hline $\begin{array}{l}\text { Baydur (2001) } \\
\text { [35] }\end{array}$ & $\begin{array}{l}74 \\
\text { Tetraplegie } \\
\text { (C3-7- } \\
\text { Verletzung): } \\
\text { 31; Paraplegie } \\
\text { (T-L4- } \\
\text { Verletzung): } 43\end{array}$ & $40 \pm 12$ & $\mathrm{SCl}$ & $\begin{array}{l}\text { Sitzend } \\
\text { Rückenlage }\end{array}$ & FVC, FEV 1 & FVC, FEV $1: p>0,05$ zwischen den Positionen \\
\hline $\begin{array}{l}\text { Ben Dov (2009) } \\
\text { [17] }\end{array}$ & 12 & $42 \pm 11$ & $\begin{array}{l}\text { Neurologisch } \\
\text { stabil } \\
\text { Tetraplegie C5-8 }\end{array}$ & $\begin{array}{l}\text { Sitzend } \\
\text { Rückenlage }\end{array}$ & FVC & FVC: Sitzend < Rückenlage \\
\hline Park (2010) [34] & 43 & $35,0 \pm 12,6$ & $\begin{array}{l}\mathrm{SCl} \\
\mathrm{C} 6-\mathrm{C} 8\end{array}$ & $\begin{array}{l}\text { Sitzend } \\
\text { Rückenlage }\end{array}$ & FVC & FVC: Sitzend < Rückenlage \\
\hline $\operatorname{Kim}(2012)[36]$ & $\begin{array}{l}45 \\
\text { Zervikal: } 15 \\
\text { Thorakal: } 13 \\
\text { Lumbal: } 17\end{array}$ & $\begin{array}{l}\text { Zervikal } \\
43,2 \pm 1,3 \\
\text { Thorakal } \\
49,8 \pm 4,9 \\
\text { Lumbal } \\
52,2 \pm 4,4\end{array}$ & $\mathrm{SCl}$ & $\begin{array}{l}\text { Sitzend } \\
\text { Rückenlage }\end{array}$ & FVC, FEV & 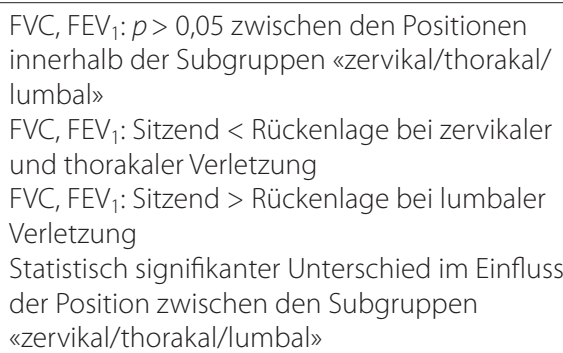 \\
\hline
\end{tabular}


Tab. 2 (Fortsetzung)

\begin{tabular}{|c|c|c|c|c|c|c|}
\hline Erstautor (Jahr) & $\begin{array}{l}\text { Teilnehmer- } \\
\text { zahl }\end{array}$ & $\begin{array}{l}\text { Alter } \\
\text { (Jahre) }\end{array}$ & $\begin{array}{l}\text { Patienten- } \\
\text { population }\end{array}$ & Positionen & $\begin{array}{l}\text { Lungenfunktions- } \\
\text { prüfung }\end{array}$ & Hauptergebnisse \\
\hline $\begin{array}{l}\text { Terson de } \\
\text { Paleville (2014) } \\
\text { [37] }\end{array}$ & $\begin{array}{l}27 \\
\text { Komplette } \\
\text { motorische } \\
\text { Schädigung: } \\
13 \\
\text { Inkomplette } \\
\text { motorische } \\
\text { Schädigung: } \\
14 \\
\text { Zervikal:15, } \\
\text { Thorakal: } 12\end{array}$ & $40 \pm 14$ & $\mathrm{SCl}$ & $\begin{array}{l}\text { Sitzend } \\
\text { Rückenlage }\end{array}$ & $\begin{array}{l}\text { FVC, FEV } 1, \text { PEmax, } \\
\text { PImax }\end{array}$ & $\begin{array}{l}\text { FVC, FEV } 1 \text { : } p>0,05 \text { zwischen den Positionen für } \\
\text { alle Patienten zusammen } \\
\text { FVC: Sitzend }<\text { Rückenlage bei zervikaler/ } \\
\text { kompletter motorischer Schädigung } \\
\text { FVC: Sitzend > Rückenlage bei thorakaler } \\
\text { inkompletter motorischer Schädigung } \\
\text { FEV } 1 \text { : Sitzend > Rückenlage bei inkompletter } \\
\text { motorischer Schädigung } \\
\text { FEV } 1 \text { : Sitzend > Rückenlage bei thorakaler } \\
\text { inkompletter motorischer Schädigung } \\
\text { PEmax: Sitzend > Rückenlage bei allen } \\
\text { Patienten } \\
\text { PEmax: Sitzend > Rückenlage bei kompletter } \\
\text { motorischer Schädigung } \\
\text { PEmax: Sitzend > Rückenlage bei zervikaler } \\
\text { inkompletter motorischer Schädigung } \\
\text { PImax: } p>0,05 \text { zwischen den Positionen für } \\
\text { alle Patienten zusammen } \\
\text { PImax: Sitzend > Rückenlage bei thorakaler } \\
\text { kompletter motorischer Schädigung }\end{array}$ \\
\hline $\begin{array}{l}\text { Miccinilli (2016) } \\
{[40]}\end{array}$ & $\begin{array}{l}20 \\
\text { Tetraplegie } \\
\text { (C3-7): 9; } \\
\text { Paraplegie } \\
(T 1-8): 11\end{array}$ & $\begin{array}{l}\text { Tetraplegie: } \\
29,4 \pm 10,5 \\
\text { Paraplegie: } \\
36,6 \pm 10,3\end{array}$ & $\mathrm{SCl}$ & $\begin{array}{l}\text { Sitzend } \\
\text { Rückenlage }\end{array}$ & $\mathrm{VC}, \mathrm{FEV}_{1}$ & VC, FEV 1 : Sitzend $<$ Rückenlage \\
\hline $\begin{array}{l}\text { Varrato (2001) } \\
\text { [25] }\end{array}$ & 38 & 61 & ALS & $\begin{array}{l}\text { Sitzend } \\
\text { Rückenlage }\end{array}$ & FVC & FVC: Sitzend > Rückenlage \\
\hline $\begin{array}{l}\text { Park (2010) } \\
{[34]}\end{array}$ & 45 & $54,4 \pm 11,1$ & ALS & $\begin{array}{l}\text { Sitzend } \\
\text { Rückenlage }\end{array}$ & FVC & FVC: Sitzend > Rückenlage \\
\hline $\begin{array}{l}\text { Poussel (2014) } \\
\text { [38] }\end{array}$ & 58 & $42,6 \pm 12,9$ & $\begin{array}{l}\text { Myotone } \\
\text { Dystrophie }\end{array}$ & $\begin{array}{l}\text { Sitzend } \\
\text { Rückenlage }\end{array}$ & FVC, FEV 1 & FVC, FEV 1 : Sitzend > Rückenlage \\
\hline $\begin{array}{l}\text { Watson (2005) } \\
\text { [43] }\end{array}$ & 10 & $\begin{array}{l}49 \pm 6 \\
M W \pm S E\end{array}$ & $\begin{array}{l}\text { Adipositas } \\
\text { Mittlerer BMI: } \\
44 \pm 3 \quad M W \pm S E\end{array}$ & $\begin{array}{l}\text { Sitzend } \\
\text { Rückenlage }\end{array}$ & $\begin{array}{l}\text { TLC, VC, } \\
\text { RV, FRC }\end{array}$ & $\begin{array}{l}\text { TLC, VC, RV, FRC: } p>0,05 \text { zwischen den } \\
\text { Positionen }\end{array}$ \\
\hline Razi (2007) [32] & 51 & $39,86 \pm 10,1$ & $\begin{array}{l}\text { Adipositas } \\
\text { Mittlerer BMI: } \\
36,7 \pm 4,1\end{array}$ & $\begin{array}{l}\text { Stehend } \\
\text { Sitzend }\end{array}$ & $\begin{array}{l}\mathrm{FVC}, \mathrm{FEV}_{1}, \mathrm{FEV}_{1} / \\
\mathrm{FVC}\end{array}$ & $\begin{array}{l}\mathrm{FVC}_{1} \mathrm{FEV}_{1}, \mathrm{FEV}_{1} / \mathrm{FVC}: p>0,05 \text { zwischen den } \\
\text { Positionen }\end{array}$ \\
\hline $\begin{array}{l}\text { Benedik (2009) } \\
{[52]}\end{array}$ & 32 & $\begin{array}{l}\text { Spanne: } \\
18-75\end{array}$ & $\begin{array}{l}\text { Gesunde, leichte } \\
\text { bis mäßige } \\
\text { Adipositas } \\
\text { Mittlerer BMI: } \\
32,7 \pm 3,5\end{array}$ & $\begin{array}{l}\text { Sitzend } \\
\text { Rückenlage }\end{array}$ & FRC & FRC: Sitzend > Rückenlage \\
\hline $\begin{array}{l}\text { Sebbane (2015) } \\
\text { [41] }\end{array}$ & 12 & $44 \pm 14$ & $\begin{array}{l}\text { Morbide } \\
\text { Adipositas } \\
\text { Mittlerer BMI: } \\
45 \pm 5 \\
\text { Z. n. } \\
\text { bariatrischer } \\
\text { Operation } \\
\text { Mittlerer BMI: } \\
31 \pm 5\end{array}$ & $\begin{array}{l}\text { Sitzend } \\
\text { Rückenlage }\end{array}$ & $\begin{array}{l}\text { TLC, RV, VC, FRC, } \\
\text { FEV }_{1}\end{array}$ & $\begin{array}{l}\text { FEV } 1 \text { : Sitzend }>\text { Rückenlage bei morbider } \\
\text { Adipositas } \\
\text { TLC, RV, FRC, VC: } p>0,05 \text { zwischen den } \\
\text { Positionen bei morbider Adipositas } \\
\text { FRC, FEV } V_{1} \text { : Sitzend }>\text { Rückenlage bei Z. n. } \\
\text { bariatrischer Operation } \\
\text { TLC, RV, VC: } p>0,05 \text { zwischen den Positionen } \\
\text { bei Z. n. bariatrischer Operation }\end{array}$ \\
\hline
\end{tabular}

Sofern nicht anders angegeben, handelt es sich bei allen Werten um Mittelwerte \pm SD (standard deviation, Standardabweichung). ALS: Amyotrophe Lateralsklerose; BMI: Body Mass Index; CHF: Congestive heart failure (kongestive Herzinsuffizienz); COPD: Chronic obstructive pulmonary disease (chronisch obstruktive Lungenerkrankung); $D_{L c o}$ : Diffusing capacity of the lungs for carbon monoxide (Kohlenmonoxid-Diffusions-kapazität der Lunge); $D_{L C O} / V A$ : Diffusing capacity of the lung for carbon monoxide divided by alveolar volume (Kohlenmonoxid-Diffusionskapazität der Lunge geteilt durch das Alveolarvolumen); FEV ${ }_{1}$ : forciertes exspiratorisches Volumen in 1 s; FRC: Functional residual capacity (funktionelle Residualkapazität); FVC: Forced vital capacity (forcierte Vitalkapazität); LSL: Linksseitenlage; MW: Mittelwert; PEF: Peak expiratory flow (exspiratorischer Spitzenfluss); PEmax: Maximal expiratory pressure (maximaler exspiratorischer Druck): PImax: Maximal inspiratory pressure (maximaler inspiratorischer Druck); RSL: Rechtsseitenlage; RV: Residualvolumen; SCl: Spinal cord injury (Rückenmarkverletzung); SE: standard error (Standardfehler); Z. n.: Zustand nach; TLC: Total lung capacity (totale Lungenkapazität); VC: Vital capacity (Vitalkapazität). 
Operation im Sitzen höhere FEV1-Werte auf als in Rückenlage [41]. In einer anderen Studie fand sich bei adipösen Patienten kein Unterschied in den $\mathrm{FEV}_{1}$-Werten zwischen Stehen und Sitzen [32].

\section{FEV $/$ /FVC}

In 7 Studien wurde das Verhältnis $\mathrm{FEV}_{1} / \mathrm{FVC}$ für verschiedene Körperpositionen bei gesunden Probanden verglichen [18, 19, 23, 24, 27, 28, 42]. Mehreren Studien zufolge war das FEV 1 /FVC-Verhältnis im Sitzen höher als in Rückenlage [23, 28], im Sitzen höher als in LSL [19] und im Stehen höher als in Rücklage, RSL und LSL [19]; da das $\mathrm{FEV}_{1} / \mathrm{FVC}$-Verhältnis in allen Körperpositionen jedoch $>70 \%$ betrug, war der Unterschied klinisch nicht bedeutsam. Andere Studien zeigten keinen Unterschied zwischen der sitzenden Position und der Rückenlage [18, 24, 27] oder Stehen, Sitzen und Rückenlage [42].

Bei Patienten mit Asthma, CHF und Adipositas war kein statistisch signifikanter Unterschied im Verhältnis $\mathrm{FEV}_{1} / \mathrm{FVC}$ zwischen den verschiedenen Körperhaltungen feststellbar [18, 27, 32, 42].

\section{VC}

Der Einfluss der Körperposition auf die VC wurde in 6 Studien mit gesunden Probanden untersucht [21, 24, 28, 39, 43, 44]. Die meisten Studien fanden keinen Unterschied zwischen Sitzen und Rückenlage [21, 24, 28, 43] oder zwischen Sitzen und RSL oder LSL [21]. In einer Studie [39] war die VC im Sitzen höher als in Rückenlage, während die VC in einer anderen Studie [44] in Rückenlage höher ausfiel als im Sitzen - allerdings nur bei Frauen. Patienten mit CHF wiesen in einer Studie [27] im Sitzen eine höhere VC auf als in Rückenlage, wohingegen in einer anderen Studie [21] kein statistisch signifikanter Unterschied zwischen diesen Positionen beobachtet wurde. Patienten mit Rückenmarksverletzung zeigten in Rückenlage eine höhere VC als im Sitzen [40]. Bei Patienten mit Adipositas wurde kein Unterschied in der VC zwischen der sitzenden Position und der Rückenlage berichtet [41, 43].

\section{PEF}

Dreizehn Studien beurteilten die PEF-Werte bei den unterschiedlichen Körperpositionen [3, 22-24, 31, 33, 45-51]. In 8 Studien wurden nur gesunde Erwachsene untersucht [3, 22-24, 45, 48, 50, 51], 3 Studien beurteilten gesunde Probanden und Patienten mit COPD oder Asthma [31, 46, 49], eine Studie schloss erwachsene Patienten mit zystischer Fibrose ein [47] und in einer Studie wurden Patienten mit Rückenmarksverletzung beurteilt [33]. 9 Studien, in denen die stehende oder sitzende Position mit der Rückenlage oder RSL und LSL verglichen wurde, ergaben höhere PEFWerte im Stehen und im Sitzen [3, 22-24, 31, 45-48]. In 3 von 6 Studien zum Vergleich der stehenden und sitzenden Position fanden sich höhere PEF-Werte im Stehen $[46,50,51]$ und eine Studie berichtete höhere PEF-Werte im Sitzen [22]. Höchstwahrscheinlich ist jedoch keiner dieser angegebenen Unterschiede in den PEF-Werten klinisch bedeutsam. Bei Patienten mit Rücken- marksverletzung und kompletter Tetraplegie fielen die PEF-Werte in Rückenlage um 12\% höher aus als im Sitzen [33].

\section{FRC}

Die FRC wurde in 5 Studien mittels Heliumdilution beurteilt [27, $41,43,52,53$ ]. Gesunde Probanden wiesen im Stehen [53] und im Sitzen $[27,43]$ höhere FRC-Werte auf als in Rückenlage, und die Unterschiede waren statistisch signifikant und klinisch bedeutsam. Bei Patienten mit Adipositas (mittlerer BMI: 44-45) [41, 43] oder CHF [27] erreichte der Unterschied zwischen Sitzen und Rückenlage dagegen keine statistische Signifikanz und er war bei Patienten nach bariatrischer Operation (mittlerer BMI: 31) höher [41]. Eine andere Studie [52], die Patienten mit leichter bis mäßiger Adipositas einschloss (mittlerer BMI: 32), berichtete, dass die FRC-Werte im Sitzen höher ausfielen als in Rückenlage, und der Unterschied war sowohl statistisch signifikant als auch klinisch bedeutsam.

\section{TLC}

Zwei Studien beurteilten die TLC mittels Heliumdilution bei gesunden Probanden [43] sowie Patienten mit Adipositas [41, 43] und fanden keinen statistisch signifikanten Unterschied zwischen der sitzenden Position und der Rückenlage.

RV

Zwei Studien beurteilten das RV mittels Heliumdilution bei gesunden Probanden [43] sowie Patienten mit Adipositas [41, 43] und fanden keinen statistisch signifikanten Unterschied zwischen der sitzenden Position und der Rückenlage.

\section{PEmax}

Sechs Studien untersuchten den Zusammenhang zwischen Körperposition und PEmax bei gesunden Probanden [3, 28, 39, 46, 54, 55]. Die PEmax-Werte der Probanden fielen im Stehen höher aus als in Rückenlage, im Stehen höher als im Sitzen und in RSL, im Sitzen höher als in Rückenlage [54] und im Sitzen höher als in Rückenlage und RSL [46]; allerdings waren die in diesen Studien berichteten Unterschiede klinisch nicht bedeutsam. In anderen Studien wurden keine Unterschiede in den PEmax-Werten zwischen Sitzen und Rückenlage [28, 39] oder zwischen Sitzen, Rückenlage, RSL und LSL beobachtet $[3,55]$.

Bei COPD-Patienten fanden sich höhere PEmax-Werte im Stehen oder Sitzen als in Rückenlage oder RSL [46] und Patienten mit zystischer Fibrose zeigten höhere Werte im Stehen und Sitzen als in RSL [47]. Die Unterschiede waren klinisch nicht bedeutsam.

Bei der Untersuchung von Patienten mit Rückenmarksverletzung fielen die PEmax-Werte bei allen Patienten sowie bei den Patienten mit kompletter motorischer Lähmung oder inkompletter zervikaler motorischer Lähmung im Sitzen signifikant höher aus als in Rückenlage [37].

\section{PImax}

In 2 Studien wurden bei gesunden Probanden im Sitzen bessere PImax-Werte beobachtet als in Rückenlage $[3,54]$. Andere Studi- 
en fanden dagegen keinen Unterschied im PImax-Wert im Sitzen versus Rückenlage $[28,39,55]$ oder im Sitzen versus RSL und LSL [3, 55]. Bei Patienten mit chronischer Rückenmarksverletzung war keine signifikante Veränderung der PImax-Werte zwischen Sitzen und Rückenlage nachweisbar, mit Ausnahme einer Subgruppe von Patienten mit kompletter thorakaler motorischer $\mathrm{Pa}$ rese, die eine statistisch signifikante und klinisch bedeutsame Verbesserung im Sitzen zeigten [37].

\section{$D_{L C O}$}

Sieben Studien beurteilten den Einfluss der Körperposition auf die Diffusionskapazität. 6 Studien schlossen gesunde Probanden ein $[18,20,21,24,56,57], 3$ Studien schlossen Patienten mit CHF ein $[18,21,58]$ und eine Studie schloss COPD-Patienten ein [57]. Gesunde Probanden zeigten in 2 Studien $[24,56]$ eine statistisch signifikante und klinisch bedeutsame Verbesserung der $\mathrm{D}_{\mathrm{LCO}}$ in Rückenlage versus Sitzen und in eine Studie [57] war ein Trend zu einer höheren $\mathrm{D}_{\mathrm{LCO}}$ in Rückenlage versus Sitzen zu beobachten. Dieser Unterschied erreichte jedoch keine statistische Signifikanz. In einer Studie [18] war die $\mathrm{D}_{\text {LCO }}$ im Sitzen höher als in Rückenlage, wohingegen einer andere Studie keinen Unterschied in der $D_{\text {LCO }}$ zwischen diesen Positionen ergab [21]. Eine Studie [21] berichtete höhere $\mathrm{D}_{\mathrm{LCO}}$-Werte im Sitzen versus Seitenlage, während in einer anderen Studie [20] kein Unterschied zwischen diesen Positionen feststellbar war. Bei COPD-Patienten fand sich keine statistisch signifikante Änderung der $\mathrm{D}_{\text {LCO }}$ zwischen der sitzenden Position und der Rückenlage [57].

Drei Studien untersuchten die Diffusionskapazität bei Patienten mit CHF [18, 21, 58]. Einer Studie zufolge [58] hatte der Positionswechsel von der Rückenlage zum Sitzen unterschiedliche Veränderungen der Diffusionskapazität zur Folge. Bei manchen Patienten verbesserte sich die Diffusionskapazität im Sitzen, während sich bei anderen keine Veränderung oder eine Abnahme zeigte. Im Durchschnitt bestand zwischen den beiden Positionen kein statistisch signifikanter Unterschied. Nach Ansicht der Autoren waren die unterschiedlichen Reaktionen durch Druckschwankungen im Lungenkreislauf bedingt. Eine andere Studie [18] fand keinen signifikanten Unterschied in der Diffusionskapazität zwischen der sitzenden Position und der Rückenlage. In der 3. Studie wurde eine $\mathrm{D}_{\mathrm{LCO}}$-Abnahme in Seitenlage gegenüber der sitzenden Position berichtet [21].

\section{Diskussion}

Die meisten Studien der vorliegenden systematischen Übersichtsarbeit von 43 Publikationen, in denen der Einfluss der Körperposition auf die Lungenfunktion beurteilt wurde, zeigten eine bessere Lungenfunktion bei eher aufrechten Körperhaltungen, und dies sowohl bei gesunden Probanden als auch bei Patienten mit Lungen- und Herzkrankheiten, neuromuskulären Erkrankungen und Adipositas. Bei Patienten mit Rückenmarksverletzung ist der Effekt komplexer und hängt vom Schweregrad und der Höhe der Verletzung ab. Demgegenüber steigt die mittels $\mathrm{D}_{\text {LCO }}$ gemessene Diffusionskapazität bei gesunden Probanden in Rückenlage an, während der Effekt bei CHF-Patienten den Annahmen zufolge vom Druck im Lungenkreislauf abhängt.

Die verringerte FVC in liegenden Positionen spiegelt möglicherweise zum einen das erhöhte thorakale Blutvolumen infolge der schwerkraftbedingten Erleichterung des venösen Rückstroms wider (was eher bei Patienten mit Herzinsuffizienz von Bedeutung ist), zum anderen ist sie möglicherweise durch die kraniale Zwerchfellverlagerung aufgrund des im Liegen erhöhten abdominalen Drucks bedingt (was eher bei adipösen Patienten von Bedeutung ist) [59]. In Seitenlage scheint der Effekt auf die FVC ähnlich auszufallen wie im Liegen, obgleich nur die abhängige Hälfte des Zwerchfells verlagert wird [59]. Andere Faktoren, die zu niedrigeren FVC-Werten in Seitenlage führen können, sind ein erhöhter Atemwegswiderstand und eine herabgesetzte Lungen-Compliance infolge anatomischer Unterschiede zwischen dem rechten und dem linken Lungenflügel sowie einer Verlagerung der mediastinalen Strukturen [20].

Die $\mathrm{FEV}_{1}$-Werte waren in aufrechten Positionen ebenfalls höher. Im Liegen sind das exspiratorische Volumen und der Atemfluss eingeschränkt, was möglicherweise darauf zurückzuführen ist, dass im Liegen der Atemwegswiderstand ansteigt, die elastische Rückstellung der Lunge abnimmt oder dass ein geringerer mechanischer Vorteil der forcierten Exspiration besteht, der vermutlich die großen Atemwege betrifft [20]. Bei Asthma-Patienten könnte der Anstieg der FVC im Stehen durch den erhöhten Atemwegsdurchmesser in dieser Position bedingt sein [30].

Bei Patienten mit CHF ist die Lunge unflexibel und schwer und das Herz ist vergrößert und schwer, was die negativen Auswirkungen der gegenseitigen Abhängigkeit von Herz und Lunge verstärkt [60]. Mit einem Anstieg der Herzmasse kommt es zu einer Verringerung des Volumen der Lunge, ihrer mechanischen Funktion und Diffusionskapazität [61, 62]; d.h., das Herz drückt im Sitzen auf das Zwerchfell und im Liegen auf einen Lungenflügel. Dies hat Auswirkungen auf die Ausdehnung der Lunge nach lateral, ermöglicht jedoch eine Senkung des Zwerchfells und eine Ausdehnung der Lunge nach kaudal. In Seitenlage drückt das Herz auf einen Lungenflügel und komprimiert sowohl die Atemwege als auch das Lungenparenchym, was einen Rückgang von $\mathrm{FEV}_{1}$ und FVC aufgrund der Atemwegskompression zur Folge hat [21]. Bei CHF-Patienten steigen in Rückenlage sowohl die elastische Belastung (verringerte Lungen-Compliance) als auch der Atemwegswiderstand an [63].

Änderungen der FVC beim Wechsel vom Sitzen in die Rückenlage spiegeln möglicherweise die Zwerchfellkraft/-paralyse wider. Die FVC ist daher ein wichtiges klinisches Instrument für die Beurteilung der Zwerchfellschwäche bei Patienten mit neuromuskulären Erkrankungen [64]. Bei Patienten mit ALS dient die FVC-Messung in Rückenlage zur Untersuchung der Zwerchfellschwäche [65] sowie zur Vorhersage einer Orthopnoe [25] und der Überlebensdauer [66, 67]. Die American Academy of Neurology kam zu dem Schluss, dass die FVC-Messung in Rückenlage zum Nachweis einer Zwerchfellschwäche bei ALS-Patienten vermutlich effektiver ist als die FVC-Messung in aufrechter Position und dass sie besser mit den Symptomen einer Hypoventilation korreliert [68]. 
Bei Patienten mit zervikaler Rückenmarksverletzung (Tetraplegie) steigen die FVC- und $\mathrm{FEV}_{1}$-Werte in der Rückenlage gegenüber der sitzenden Position an. In Rückenlage nimmt die inspiratorische Atemexkursion des Zwerchfells zu, weil die Muskelfasern am Ende der Exspiration länger sind und an einem effektiveren Punkt ihrer Längen-Spannungs-Kurve arbeiten [6971]. Dieser Mechanismus ist besonders bei Patienten von Bedeutung, bei denen das Zwerchfell die Hauptatemarbeit leistet, da ihre Intercostal- und Bauchmuskulatur infolge der Rückenmarksverletzung inaktiv ist.

Berichten zufolge fällt die FRC in aufrechter Position bei gesunden Probanden $[27,43,53]$ und bei Patienten mit leichter bis mäßiger Adipositas höher aus [41, 52]. Beim Wechsel von der Rückenlage in eine aufrechte Position steigt die FRC aufgrund des verminderten pulmonalen Blutvolumens und der Absenkung des Zwerchfells an. Dadurch verschiebt sich der Punkt der Volumen-DruckKurve, an dem die Ruheatmung erfolgt, was eine erhöhte LungenCompliance zur Folge hat, und eine identische Druckänderung würde daher - bei gleich bleibendem Atemantrieb - ein größeres Inspirationsvolumen bewirken [53]. Berichten zufolge sind jedoch bei CHF-Patienten keine Änderungen der FRC beim Wechsel zwischen Sitzen und Rückenlage zu beobachten [27]. Bei Herzinsuffizienz kann die verminderte Lungen-Compliance in Rückenlage die passive Änderung des Lungenvolumens verringern, doch wird die FRC durch eine mögliche Anpassung der Atemmuskelaktivität oder der glottalen Muskelaktivität über dem Relaxationsvolumen gehalten [27]. Bei adipösen Patienten fiel die FRC im Sitzen niedriger aus als bei gesunden Probanden, wobei es jedoch in Rückenlage zu keinem weiteren Rückgang kam [43].

Untersuchungen mit gesunden Probanden [3, 23, 24, 46, 48, 50, 51] und Patienten mit Lungenerkrankungen [31, 46, 47] ergaben höhere Werte für PEF, PEmax und PImax in aufrechter Position. Dies könnte durch Veränderungen des Lungenvolumens in den verschiedenen Positionen bedingt sein.

Es hat sich gezeigt, dass das Lungenvolumen im Stehen und Sitzen am höchsten ist $[72,73]$. Bei höheren Lungenvolumina sind die elastischen Rückstellkräfte von Lunge und Thoraxwand größer. Außerdem befinden sich die Ausatemmuskeln in einem günstigeren Bereich der Längen-Spannungs-Kurve und sind daher in der Lage, einen höheren intrathorakalen Druck aufzubauen. Dadurch werden potenziell höhere exspiratorische Drücke erzeugt, die die Atemluft mit hoher Geschwindigkeit durch die engen Atemwege drücken, sodass es zu höheren PEmax-, PEF- und $\mathrm{FEV}_{1}$-Werten kommt. Mit abnehmendem Lungenvolumen wird die Muskellänge ungünstiger, was zur Folge hat, dass die PEmaxWerte im Sitzen niedriger ausfallen als im Stehen und im Liegen sogar noch niedriger. Die PEmax-Änderung hat Auswirkungen auf die PEF-Werte [46].

Im Stehen zieht die Schwerkraft die mediastinalen und abdominalen Strukturen nach unten, sodass mehr Raum in der Thoraxhöhle entsteht, was eine weitere Ausdehnung der Lunge und gröBere Lungenvolumina ermöglicht [74]. Dadurch und durch die verminderte Kompression der basalen Lungenabschnitte erfolgt eine alveoläre Rekrutierung und die Lungen-Compliance steigt.
Die inspiratorischen Muskeln können sich noch weiter ausdehnen, sodass sich das Zwerchfell weiter nach unten kontrahieren kann und das Lungenvolumen steigt [46].

Im Sitzen fallen das Lungenvolumen im Vergleich zum Stehen häufig etwas niedriger aus. Dies lässt sich durch verschiedene Mechanismen erklären. Zum einen liegen die Bauchorgane im Sitzen weiter oben und behindern dadurch die Zwerchfellbewegung, sodass eine geringere Inspiration möglich ist. Zum anderen befinden sich die Bauchmuskeln an einem ungünstigeren Punkt der Längen-Spannungs-Kurve, da die Kombination aus Hüftbeugung und höherer Lage des Bauchinhalts einen Druck nach oben ausübt. Und zum dritten kann die Rückenlehne des Stuhls die Ausdehnung des Brustkorbs behindern. Diese 3 Faktoren erklären die etwas niedrigeren PEmax- und PEF-Werte im Sitzen versus Stehen [46].

Die Rückenlage wirkt sich negativ auf die Zwerchfellkraft aus und das intrathorakale Blutvolumen steigt, was dazu führt, dass die PEmax- und PEF-Werte in Rückenlage vermindert sind [3].

In Seitenlage (RSL oder LSL) und bei flacher Unterlage fällt der Bauchinhalt nach vorne. Dadurch wird die abhängige Zwerchfellhälfte auf eine für den Spannungsaufbau günstige Länge gedehnt, während die nicht abhängige Zwerchfellhälfte mehr abgeflacht ist. Auf diese Weise können sich Änderungen der Lungenvolumina durch eine bessere Zwerchfellkontraktion einerseits, jedoch verminderten Thoraxraum andererseits gegenseitig ausgleichen [46]. Der in Rückenlage beobachtete verringerte PImax könnte durch eine Überlastung des Zwerchfells infolge der Verlagerung des Bauchinhalts während der maximalen inspiratorischen Atemarbeit bedingt sein, die die günstigere Position des Zwerchfells auf der Längen-Spannungs-Kurve aufhebt. Zudem ist die Länge aller anderen Einatemmuskeln in Rückenlage weniger günstig [75].

Bei Patienten mit zervikaler Rückenmarksverletzung und hoher Tetraplegie fielen die PEF-Werte in Rückenlage höher aus als im Sitzen [33], ebenso wie die FVC- und FEV ${ }_{1}$-Werte.

Gesunde Probanden zeigten in den meisten Studien eine höhere $\mathrm{D}_{\text {LCO }}$ in Rückenlage als im Sitzen [24, 56, 57]. Diese Verbesserung wird dem mäßigen Anstieg des alveolären Blutvolumens in Rückenlage zugeschrieben, der durch eine Rekrutierung von Lungenkapillaren beim Wechsel von einer aufrechten Position in die Rückenlage bedingt ist. Mit zunehmendem Alter kann dieser Anstieg abnehmen [76]. Dies könnte erklären, weshalb in einer Studie, die Teilnehmer mit einem Durchschnittsalter von 61 Jahren einschloss [21], kein Unterschied in den $\mathrm{D}_{\mathrm{LCO}}$-Werten zwischen Sitzen und Rückenlage beobachtet wurde.

In Seitenlage drückt das Herz auf einen Lungenflügel, wodurch sowohl die Atemwege als auch das Lungenparenchym zusammengedrückt werden. Dies hat eine Verringerung des alveolären Blutvolumens zur Folge, sodass ein Missverhältnis zwischen Ventilation und Perfusion entsteht. Durch diese Effekte kommt es zu einem Rückgang der Diffusionskapazität in Seitenlage [21].

Bei COPD-Patienten war keine Veränderung der $\mathrm{D}_{\mathrm{LCO}}$ zwischen Sitzen und Rückenlage nachweisbar [57], was durch eine verringerte FVC und eine Schädigung der Alveolen bei diesen Patienten bedingt sein könnte. Diese Effekte können negative Auswirkun- 
gen auf die Diffusionskapazität haben und stehen dem positiven Effekt des Anstiegs des alveolären Blutvolumens gegenüber [57]. Bei Patienten mit CHF wurden verschiedene Muster des Effekts der Körperhaltung auf die $\mathrm{D}_{\text {LCO }}$ beobachtet [58]. Die Änderung der $D_{\text {LCO }}$ war vermutlich durch die Änderung des alveolären Blutvolumens bedingt, höchstwahrscheinlich infolge von Unterschieden im Druck der Lungenarterien und in den Herzmaßen [58].

\section{Einschränkungen der Studie}

Die vorliegende Übersichtsarbeit weist einige Einschränkungen auf. Zum einen ist der Evidenzgrad der Studien relativ niedrig. Aufgrund der Natur der untersuchten Studienpopulationen und der verwendeten Interventionen ist es bei dieser Art von Forschungsarbeit jedoch nicht möglich, eine randomisierte kontrollierte Studie durchzuführen. Zum anderen wiesen die meisten Studien geringe Teilnehmerzahlen auf und alle Studien verwendeten ein konsekutives, willkürliches oder freiwilliges Stichprobenverfahren. Da diese Übersichtsarbeit nur Studien mit erwachsenen Probanden einschloss, ist eine Verallgemeinerung der Ergebnisse auf Kinder und Jugendliche nicht möglich. Außerdem unterschieden sich die Studienprotokolle der Studien und oftmals fehlten genauere Informationen zu den Studienprotokollen. Die Ergebnisse der LFP werden maßgeblich durch die Mitarbeit des Patienten beeinflusst. Dies könnte die in einigen Fällen beobachteten widersprüchlichen Ergebnisse erklären. Studien, die Probanden über 60 Jahre einschlossen, enthielten keine Angaben zur kognitiven Funktionsfähigkeit der Teilnehmer, ein Faktor, der möglicherweise Auswirkungen auf die Mitarbeit des Patienten hat.

Weitere Untersuchungen in diesem Bereich sind erforderlich, darunter auch Studien, die darauf ausgerichtet sind, die Lungenfunktion bei einer großen Zahl gesunder Probanden sowie Patienten mit unterschiedlichen Erkrankungen zu beurteilen. Darüber hinaus ist die Verwendung eines standardisierten Studienprotokolls notwendig, das eine Randomisierung der Körperhaltungen und der Zeitabstände zwischen den Tests (z.B. für die Auswaschung der inhalierten Gase oder eine Umverteilung des Blutvolumens) in den verschiedenen Positionen beinhaltet, um eine bessere Vergleichbarkeit der Ergebnisse zu erreichen.

\section{Schlussfolgerungen}

Bei der Durchführung der LFP ist die Körperposition von Bedeutung, da sie die Testergebnisse beeinflusst. Wie diese Übersichtsarbeit zeigt, kann eine Änderung der Körperposition je nach Patientenpopulation unterschiedliche Implikationen haben. Die Leitlinien der ATS [2] empfehlen, die LFP im Sitzen oder im Stehen durchzuführen, wobei die sitzende Position jedoch meistens bevorzugt wird. Die Festlegung der geschlechts- und altersspezifischen Normwerte für die LFP erfolgte mithilfe von Tests, die in der sitzenden Position durchgeführt wurden. Nach den Ergebnissen dieser Übersichtsarbeit ist sitzend die bevorzugte Testposition. Kliniker sollten jedoch auch andere Positionen für die Durchführung der LFP bei ausgewählten Patienten in Betracht ziehen. Bei Patienten mit Rückenmarksverletzung kann die zusätzliche
Testung in Rückenlage wichtige Informationen liefern. Bei Patienten mit neuromuskulären Erkrankungen kann die Durchführung der LFP in Rückenlage helfen, die Zwerchfellfunktion zu beurteilen.

Die Körperposition ist für das Erreichen einer maximalen Atemfunktion bei der Behandlung von Patienten mit unterschiedlichen Problemen und Erkrankungen von großer Bedeutung, und es ist wichtig, die Auswirkungen der einzelnen Positionen auf das respiratorische System eines bestimmten Patienten zu kennen. Das Wissen über den Einfluss der Körperhaltung kann Ärzten und medizinischem Fachpersonal helfen, ein besseres Verständnis für die optimalen Positionen von Patienten mit unterschiedlichen Erkrankungen zu entwickeln.

\section{Hinweise}

S.K. und N.A. haben zu gleichen Teilen zur Erstellung dieser Arbeit beigetragen.

\section{Danksagung}

Die Autoren danken Prof. Ora Paltiel, Fachärztin für Innere Medizin, Hämatologie und Onkologie und Doktor der Epidemiologie und Biostatistik, für ihre wertvolle Hilfe bei der Auswahl der optimalen Instrumente zur Bewertung der Evidenzqualität und des Bias-Risikos der in diese systematische Übersichtsarbeit eingeschlossenen Studien.

Die Autoren danken Shifra Fraifeld, die als Medical Writer und medizinische Redakteurin am Krankenhaus arbeitet, für ihre redaktionellen Beiträge während der Erstellung des Manuskripts.

\section{Autorenbeiträge}

S.K., E.-L.M, N.A. und A.R. waren an der Entwicklung des Studienkonzepts und -designs beteiligt. S.K., E.-L.M., N.A., A.R. und Y.Z. waren an der Datenerhebung und -analyse sowie an der Interpretation der Daten beteiligt. Die primäre Literatursuche wurde von S.K. und E.-L.M. durchgeführt. S.K. und E.-L.M. erstellten einen Manuskriptentwurf. S.K., E.L.M., N.A., A.R. und Y.Z. nahmen eine kritische Überprüfung und Überarbeitung der wissenschaftlichen Inhalte des Manuskripts vor. Alle Autoren überprüften die finale Version des Manuskripts vor der Einreichung und alle übernehmen Verantwortung für die Integrität des wissenschaftlichen Vorgehens und der Ergebnisse. Alle Autoren haben die finale Fassung des Manuskripts gelesen und freigegeben.

\section{Genehmigung durch die Ethikkommission und Einwilligung zur Teilnahme}

Nicht zutreffend - systematische Übersichtsarbeit.

\section{Zustimmung zur Veröffentlichung}

Nicht zutreffend.

\section{Disclosure Statement}

Die Autoren erklären, dass keine Interessenskonflikte bestehen. 


\section{Lizenzangabe}

Shikma Katz, Nissim Arish, Ariel Rokach, Yacov Zaltzman, Esther-Lee Marcus: The effect of body position on pulmonary function: a systematic review. BMC Pulm Med 2018;18:159 (https://doi.org/10.1186/s12890-018 0723-4). (C) The Author(s) 2018 (Übersetzung, «Publisher’s note» gekürzt), lizensiert unter CC BY 4.0 (https://creativecommons.org/licenses/by/4.0/ deed.de).

\section{Literatur}

Die Literatur ist als Supplemental Material unter www.karger.com/ ?DOI=499746 abrufbar.

\section{Zusatzmaterial}

Tabelle S1: Bewertung der in die systematische Übersichtsarbeit eingeschlossenen Publikationen gemäß Quality Assessment Tool for Before-After (Pre-Post) Studies with No Control Group des National Heart, Lung and Blood Institute [3, 15-31, 33-58].

Tabelle S2: Statistisch signifikante Unterschiede in der Lungenfunktion zwischen den verschiedenen Körperpositionen [3, 17-28, 30, 31, 33, 34, 37$41,43-48,50-54,56]$

Beide Tabellen sind als Supplemental Material unter www.karger.com/ ?DOI=499746 abrufbar. 